\title{
Cochlear implants: Current designs and future possibilities
}

\author{
Blake S. Wilson, ${ }^{1 *}$ Michael F. Dorman $^{2}$ \\ ${ }^{1}$ Department of Surgery, Division of Otolaryngology, Head \& Neck Surgery, Duke University Medical Center, Durham, \\ NC; ${ }^{2}$ Department of Speech and Hearing Science, Arizona State University, Tempe, AZ
}

\begin{abstract}
The cochlear implant is the most successful of all neural prostheses developed to date. It is the most effective prosthesis in terms of restoration of function, and the people who have received a cochlear implant outnumber the recipients of other types of neural prostheses by orders of magnitude. The primary purpose of this article is to provide an overview of contemporary cochlear implants from the perspective of two designers of implant systems. That perspective includes the anatomical situation presented by the deaf cochlea and how the different parts of an implant system (including the user's brain) must work together to produce the best results. In particular, we present the design considerations just mentioned and then describe in detail how the current levels of performance have been achieved. We also describe two recent advances in implant design and performance. In concluding sections, we first present strengths and limitations of present systems and then offer some possibilities for further improvements in this technology. In all, remarkable progress has been made in the development of cochlear implants but much room still remains for improvements, especially for patients presently at the low end of the performance spectrum.
\end{abstract}

Key words: auditory prosthesis, cochlea, cochlear implant, cortical plasticity, deafness, hearing, neural prosthesis, rehabilitation, speech perception, speech processor.

\section{INTRODUCTION}

Cochlear implants are among the great success stories of modern medicine. Thirty years ago these devices provided little more than a sensation of sound and sound cadences-they were useful as an aid to lip-reading. In the 1980s, however, systems with multiple channels of processing and multiple sites of stimulation in the cochlea were developed and these systems supported significantly higher levels of speech reception than their single-channel and single-site predecessors. In the late 1980s and continuing to the present, new and better processing strategies, in conjunction with multielectrode implants, have produced further large improvements. Indeed, a principal conclusion of the 1995 National Institutes of Health (NIH) Consensus Conference on Cochlear Implants in Adults and Children [1] was that "A majority of those individuals with the latest speech processors for their implants will score above 80 percent correct on highcontext sentences, even without visual cues.” This level of performance is remarkable and is far greater than that achieved to date with any other type of neural prosthesis.

\footnotetext{
Abbreviations: $\mathrm{ACE}=$ advanced combination encoder, $\mathrm{AzBio}=$ Arizona Biomedical (sentences), BM = basilar membrane, CIS = continuous interleaved sampling, $\mathrm{CNC}=$ consonant-nucleusconsonant, CNS $=$ central nervous system, CUNY $=$ City University of New York, EAS = electric and acoustic stimulation, $\mathrm{F} 0=$ fundamental frequency, FS = fine structure, FSP = fine structure processing, HiRes $=$ HiResolution, HiRes $120=$ HiRes with Fidelity 120 option, HL = hearing level, IHC = inner hair cell, ITD = interaural time delay, NIH = National Institutes of Health, $\mathrm{OHC}=$ outer hair cell, $\mathrm{PET}=$ positron emission tomography, $\mathrm{PP} / \mathrm{CIS}=$ peak picker/CIS, S/B = speech-to-babble ratio, $\mathrm{S} / \mathrm{N}=$ speech-to-noise ratio, SPEAK = spectral peak, $\mathrm{ST}=$ scala tympani, VCIS = virtual channel interleaved sampling.

* Address all correspondence to Prof Blake S. Wilson, 2511 Old Cornwallis Rd, Suite 100, Durham, NC 27713; 919-3143006; fax: 919-484-9229. Email: blake.wilson@duke.edu

DOI: 10.1682/JRRD.2007.10.0173
} 
Detailed reviews of the history of cochlear implants are presented elsewhere [2-5]. Comparisons with other types of neural prostheses are presented in Wilson and Dorman, along with a discussion of how the positive experience with cochlear implants might inform the development or further development of neural prostheses for other senses, e.g., those for vision or balance [5].

The present article provides an overview of contemporary cochlear implants from the perspective of two designers of implant systems. That perspective includes the anatomical situation presented by the deaf cochlea and how the different parts of an implant system (including the user's brain) must work together to produce the best possible results. Although the emphasis is on the design and performance of processing strategies for implants, we also describe the other parts of the system so that the reader may appreciate more completely the complexity of the overall system and how the parts may interact.

This article is organized into sections that present (1) the design considerations just mentioned, (2) a review of performance with present-day implant systems, (3) strengths and limitations of those systems, (4) two recent advances in implant design and performance, and (5) possibilities for further improvements. This is an exciting time in the development of cochlear implants, with tremendous progress to date, but also with great room and excellent opportunities for further progress, especially for patients presently at the low end of the performance spectrum.

\section{DESIGN OF COCHLEAR IMPLANTS}

\section{Aspects of Normal Hearing}

In normal hearing, sound waves traveling through air reach the tympanic membrane via the ear canal, causing vibrations that move the three small bones of the middle ear. This action produces a piston-like movement of the stapes, the third bone in the chain. The "footplate" of the stapes is attached to a flexible membrane in the bony shell of the cochlea called the oval window. Inward and outward movements of this membrane induce pressure oscillations in the cochlear fluids, which in turn initiate a traveling wave of displacement along the basilar membrane (BM), a highly specialized structure that divides the cochlea along its length. This membrane has graded mechanical properties. At the base of the cochlea, near the stapes and oval window, it is narrow and stiff. At the other end, near the apex, the membrane is wide and flexible. These properties give rise to the traveling wave and to points of maximal response according to the frequency or frequencies of the pressure oscillations in the cochlear fluids. The traveling wave propagates from the base to the apex. For an oscillation with a single frequency, the magnitude of displacements increases up to a particular point along the membrane and then drops precipitously thereafter. High frequencies produce maxima near the base of the cochlea, whereas low frequencies produce maxima near the apex.

Motion of the BM is sensed by the sensory hair cells in the cochlea, which are attached to the top of the BM in a matrix of cells called the organ of Corti. The cells are arranged in four rows along the length of the cochlea. The cells in the innermost row (closest to the modiolus or "core" of the cochlea) are called the inner hair cells (IHCs), and the cells in the remaining rows are called the outer hair cells (OHCs). Each hair cell has fine rods of protein, called stereocilia, emerging from one end. When the BM moves at the location of a hair cell, the rods are deflected as if hinged at their bases. Such deflections in one direction increase the release of chemical transmitter substance at the base (other end) of the IHCs, and deflections in the other direction inhibit the release. In contrast, deflections of the stereocilia of the OHCs produce electromotile changes in the length of the cells, which in turn increase the sensitivity and sharpen the "tuning" of the $\mathrm{BM}$ to frequencies that correspond closely to the position(s) of the stimulated cells. Thus, the OHCs act as a (highly selective) biological amplifier.

The increases in chemical transmitter substance at the bases of the IHCs increase discharge activity in the immediately adjacent auditory neurons, whereas decrements in the substance inhibit activity. Changes in neural activity thus reflect events at the BM. These changes are transmitted to the brain via the auditory nerve, the collection of all neurons that innervate the cochlea.

The steps described previously are illustrated in Figure 1(a). This figure shows a cartoon of the main anatomical structures, including the tympanic membrane, the three bones of the middle ear, the oval window, the BM, the IHCs, and the adjacent neurons of the auditory nerve (shown in light blue). (The OHCs are not shown for clarity and because they do not provide the essential link from the cochlea to the brain.) 


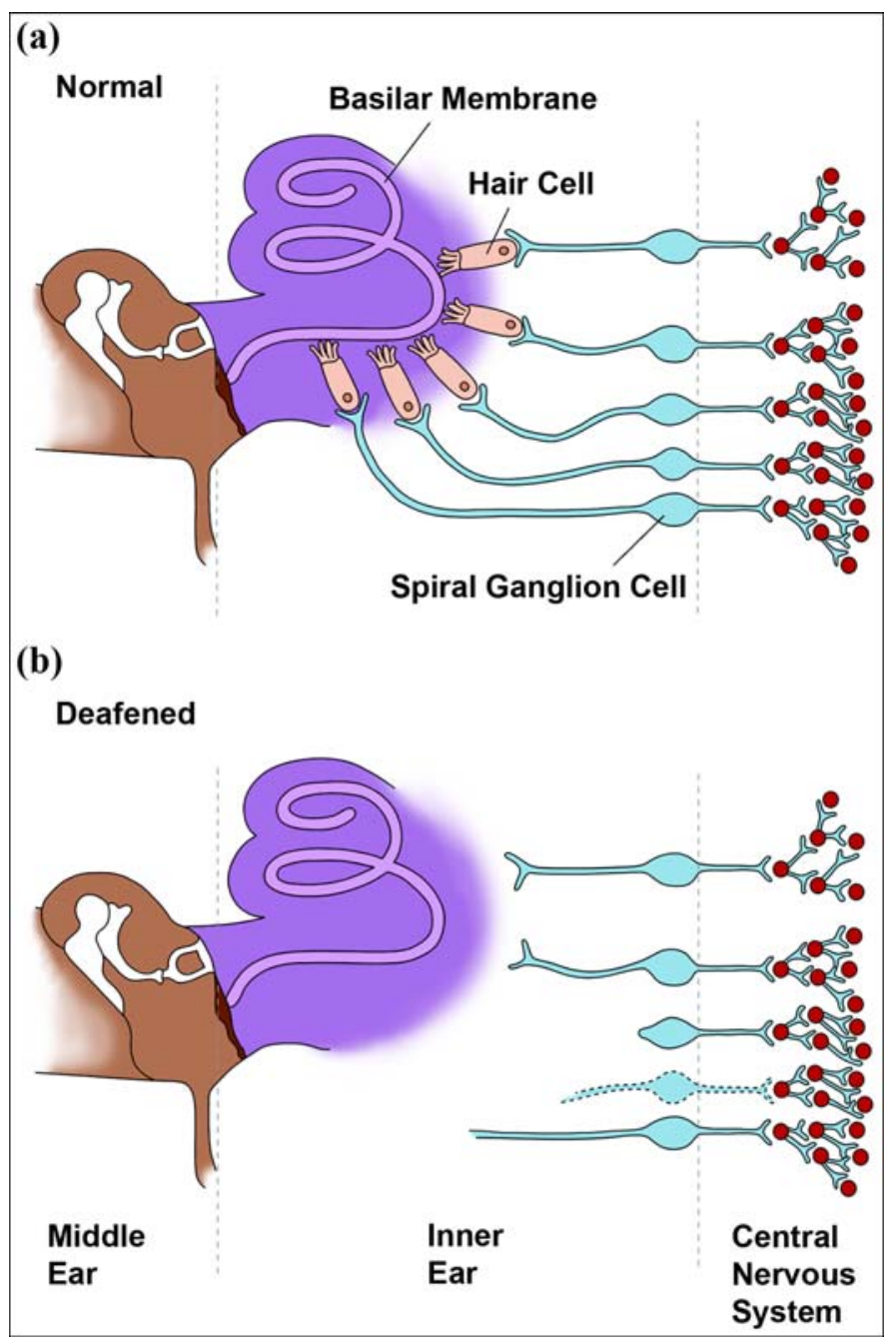

Figure 1.

Anatomical structures in (a) normal and (b) deafened ears. Note absence of sensory hair cells in (totally) deafened ear. Also note incomplete survival of spiral ganglion cells and neural processes peripheral to cells that are still viable. For simplicity, illustrations do not reflect details of structures or use consistent scale for different structures. Source: Figure is reprinted with permission from Dorman MF, Wilson BS. The design and function of cochlear implants. Am Scientist. 2004;92(5):436-45.

\section{Loss of Hearing}

The principal cause of hearing loss is damage to or complete destruction of the sensory hair cells. (Damage to or destruction of the OHCs elevates hearing thresholds and degrades frequency resolution, and damage to or destruction of the IHCs produces more profound losses up to and including total deafness.) Unfortunately, the hair cells are fragile structures and are subject to a wide variety of insults, including but not limited to genetic defects, infectious diseases (e.g., rubella and meningitis), overexposure to loud sounds, certain drugs (e.g., kanamycin, streptomycin, and cisplatin), and aging. In the deaf or deafened cochlea, the IHCs in particular are largely or completely absent, severing the connection between the peripheral and central auditory systems. The function of a cochlear prosthesis is to bypass the (missing) hair cells by directly stimulating the surviving neurons in the auditory nerve.

The anatomical situation faced by designers of cochlear implants is illustrated in Figure 1(b). The panel shows a complete absence of hair cells. In general, a small number of cells may remain for some patients, usually in the apical (low frequency) part of the cochlea. Without the normal stimulation provided by the IHCs, the peripheral parts of the neurons-between the cell bodies in the spiral ganglion and the terminals within the organ of Corti-undergo "retrograde degeneration" and eventually cease to function [6]. Fortunately, the cell bodies are far more robust. At least some usually survive, even after prolonged deafness or virulent etiologies such as meningitis [6-8]. These cells, or more specifically the nodes of Ranvier just distal or proximal to them, are the putative sites of excitation for cochlear implants.

\section{Direct Electrical Stimulation of Cochlear Neurons}

Direct stimulation of the auditory nerve is produced by currents delivered through electrodes placed in the scala tympani (ST), one of three fluid-filled chambers along the length of the cochlea. (The boundary between the ST and the scala media is formed by the BM and organ of Corti, and the boundary between the scala media and scala vestibuli is formed by Reissner's membrane.) A cutaway drawing of the implanted cochlea is presented in Figure 2. The figure shows the three chambers (in the cross sections) and a partial insertion of an electrode array into the ST. The array is inserted through a drilled opening made by the surgeon in the bony shell of the cochlea overlying the ST and close to the base of the cochlea (called a “cochleostomy”). Alternatively, the array may be inserted through the second flexible membrane of the cochlea, the round window membrane, which also is close to the basal end of the cochlea and ST (Figure 2; also note that the cochleostomy offers a "straighter shot" into the ST than the round window approach).

The depth of insertion is limited by the decreasing lumen of the ST from base to apex, the curvature of the cochlear spiral, and an uneven and unsmooth lumen, 


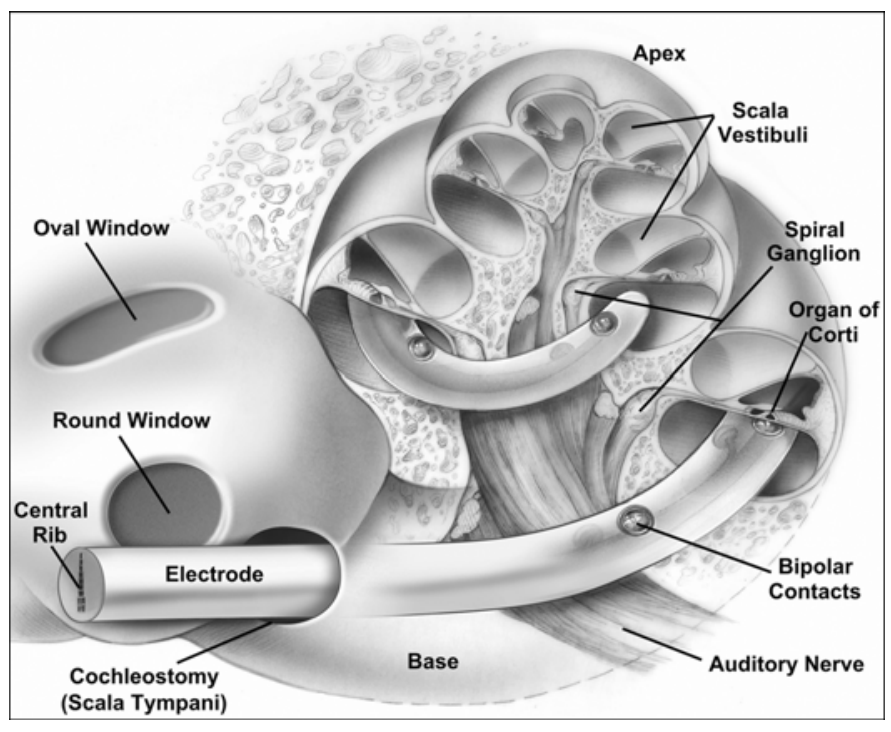

Figure 2.

Cutaway drawing of implanted cochlea. Electrode array developed at University of California at San Francisco (UCSF) is illustrated (Array detailed in Loeb GE, Byers CL, Rebscher SJ, Casey DE, Fong MM, Schindler RA, Gray RF, Merzenich MM. Design and fabrication of an experimental cochlear prosthesis. Med Biol Eng Comput. 1983;21(3):241-54. [PMID: 6688284]). Array includes eight pairs of bipolar electrodes, spaced at $2 \mathrm{~mm}$ intervals, with electrodes in each pair oriented in "offset radial" arrangement with respect to neural processes peripheral to ganglion cells in intact cochlea. Only four bipolar pairs are visible in drawing, as others are "hidden" by cochlear structures. This array was used in UCSF/Storz and Clarion ${ }^{\circledR}$ 1.0 devices. Source: Figure is reprinted with kind permission of Springer Science+Business Media from Leake PA, Rebscher SJ. Anatomical considerations and long-term effects of electrical stimulation. In: Zeng FG, Popper AN, Fay RR, editors. Auditory prostheses: Cochlear implants and beyond. New York (NY): Springer-Verlag; 2004. p. 101-48.

particularly in the apical region. No array has been inserted farther than about $30 \mathrm{~mm}$, and typical insertions are much less than that, e.g., 18 to $26 \mathrm{~mm}$. (The total length of the typical human cochlea is about $35 \mathrm{~mm}$.) In some cases, only shallow insertions are possible, such as when bony obstructions in the lumen impede further insertion.

Different electrodes in the implanted array may stimulate different subpopulations of neurons. As described previously, neurons at different positions along the length of the cochlea respond to different frequencies of acoustic stimulation in normal hearing. Implant systems attempt to mimic or reproduce this "tonotopic" encoding by stimulating basally situated electrodes (first turn of the cochlea and lower part of Figure 2) to indicate the presence of high-frequency sounds and by stimulating electrodes at more apical positions (deeper into the ST and ascending along the first and second turns in Figure 2) to indicate the presence of sounds with lower frequencies. Closely spaced pairs of bipolar electrodes are illustrated here, but arrays of single electrodes that are each referenced to a remote electrode outside the cochlea may also be used. This latter arrangement is called a "monopolar coupling configuration" and is used in all present-day implant systems that are widely applied worldwide. (The monopolar coupling is used primarily because it supports performance that is at least as good as bipolar coupling and, further, requires substantially less current and battery power to produce auditory percepts.)

The spatial specificity of stimulation with an ST electrode most likely depends on multiple factors, including the orientation and geometric arrangement of the electrodes, the proximity of the electrodes to the target neural structures, and the condition of the implanted cochlea in terms of nerve survival and ossification. An important goal of electrode design is to maximize the number of largely nonoverlapping populations of neurons that can be addressed with the electrode array. Present evidence suggests, however, that no more than 4 to 8 independent sites are available with current designs, even for arrays with as many as 22 electrodes [9-14]. Most likely, the number of independent sites is limited by substantial overlaps in the electric fields from adjacent (and more distant) electrodes. The overlaps are unavoidable for electrode placements in the ST because the electrodes are "sitting" in the highly conductive fluid of the perilymph and, additionally, are relatively far away from the target neural tissue in the spiral ganglion. A closer apposition of the electrodes to the inner wall of the ST would move them a bit closer to the target cells (Figure 2), and such placements have been shown in some cases to produce an improvement in the spatial specificity of stimulation [15]. However, a large gain in the number of independent sites may well require a fundamentally new type of electrode or a fundamentally different placement of electrodes. The many issues related to electrode design, along with prospects for the future, are discussed elsewhere [15-25].

Figure 2 shows a complete presence of hair cells (in the labeled organ of Corti) and a pristine survival of cochlear neurons. However, the number of hair cells is zero or close to it in cases of total deafness. In addition, survival of neural processes peripheral to the ganglion cells (the "dendrites") is rare in the deafened cochlea, as noted previously. Survival of the ganglion cells and central 
processes (the axons) ranges from sparse to substantial. The pattern of survival is in general not uniform, with reduced or sharply reduced counts of cells in certain regions of the cochlea. In all, the neural substrate or target for a cochlear implant can be quite different from one patient to the next. A detailed review of these observations and issues is presented in Leake and Rebscher [8].

\section{Components of Cochlear Implant Systems}

The essential components in a cochlear prosthesis system are illustrated in Figure 3 and include (1) a microphone for sensing sound in the environment, (2) a speech processor to transform the microphone input into a set of stimuli for the implanted array of electrodes, (3) a transcutaneous link for the transmission of power and stimulus information across the skin, (4) an implanted receiver/ stimulator to decode the information received from the radio frequency signal produced by an external transmitting coil and generate stimuli using the instructions obtained from the decoded information, (5) a cable to connect the outputs of the receiver/stimulator to the electrodes, and (6) the array of electrodes. These components must work together as a system to support excellent performance, and a weakness in a component can degrade performance significantly. For example, a limitation in the data bandwidth of the transcutaneous link can restrict the types and rates of stimuli that can be specified by the external speech processor, and this in turn can limit performance. A thorough discussion of considerations for the design of cochlear prostheses and their constituent parts is presented in Wilson [22].

One "component" that is not illustrated in Figure $\mathbf{3}$ is the biological component central to the auditory nerve (colored yellow in the figure), which includes the auditory pathways in the brain stem and the auditory cortices of the implant recipient. As will be described later in this article, this biological component varies in its functional integrity and capabilities across patients and is at least as important as the other parts in determining outcomes with implants.

\section{Transformation of Microphone Inputs into Stimuli for Cochlear Implants}

An important aspect of the design for any type of sensory neural prosthesis is how to transform an input from a sensor or array of sensors into a set of stimuli that can be interpreted by the nervous system. The stimuli can be electrical or tactile, for example, and usually involve multiple stimulation sites, corresponding to the spatial mapping of inputs and representations of those inputs in the nervous system. One approach to the transformation-and probably the most effective approach-is to

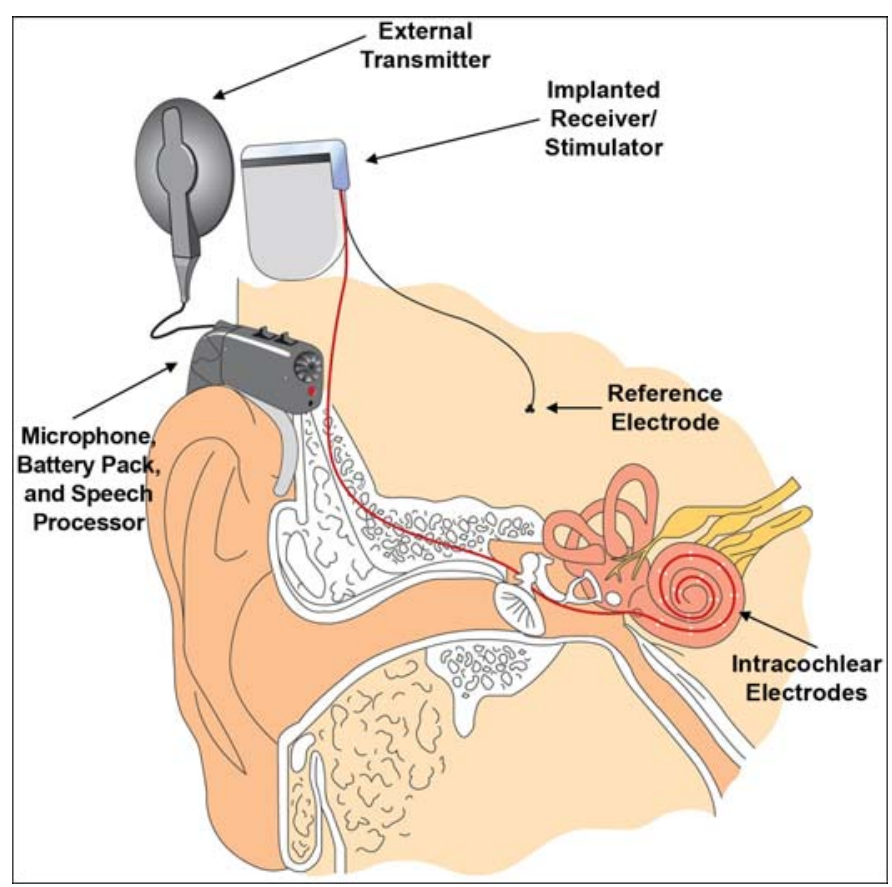

Figure 3.

Components of cochlear implant systems. TEMPO+ system (MED-EL Medical Electronics GmbH; Innsbruck, Austria) is illustrated, but all present-day implant systems share same basic components. Microphone, battery pack, and speech processor are incorporated into behind-the-ear (BTE) housing in illustrated system, much like BTEs of hearing aids. Thin cable connects output of speech processor (radio frequency signal with encoded stimulus information) to external transmitting coil that is positioned opposite implanted receiver/stimulator. Transmitting coil is held in place with pair of magnets, one in center of coil and other in implanted receiver/stimulator. Receiver/stimulator is implanted in flattened or recessed portion of skull, posterior to and slightly above pinna. Reference (or "ground") electrode is implanted at location remote from cochlea, usually in temporalis muscle. For some implant systems, metallic band around outside of receiver/stimulator package serves as reference electrode. Array of active electrodes is inserted into scala tympani through round window membrane or through larger drilled opening in bony shell of cochlea (cochleostomy) near round window. In current practice, cochleostomy is used for great majority of implant operations, although interest in round window approach is growing with recent demonstrations that this approach may help preserve any residual hearing in implanted cochlea (Skarzynski H, Lorens A, Piotrowska A, Anderson I. Preservation of low frequency hearing in partial deafness cochlear implantation (PDCI) using the round window surgical approach. Acta Otolaryngol. 2007;127(1): 41-48. [PMID: 17364328]). Figure courtesy of MED-EL Medical Electronics $\mathrm{GmbH}$. 
mimic or replicate at least to some extent the damaged or missing physiological functions that are bypassed or replaced by the prosthesis.

Of course, limitations in other parts of the prosthesis system may restrict what can be done with the transformation. Effects of limitations in the bandwidth of the transcutaneous link for cochlear implant systems have been mentioned. Also, a lack of independence among stimulus sites can greatly reduce the number of channels of information that can be conveyed to the nervous system. In such cases, a high number of channels in processing the input(s) from the sensor(s) would not in general produce any benefit and might even degrade performance.

For cochlear implants, this part of the design is called the processing strategy. As noted previously, advances in processing strategies have produced quite large improvements in the speech reception performance of implant patients, from recognition of a tiny percentage of monosyllabic words with the first strategies that used multiple processing channels and multiple sites of stimulation in the cochlea, for example, to recognition of a high percentage of monosyllabic words with the current strategies.
One of the simpler and most effective approaches for representing speech and other sounds with present-day cochlear implants is illustrated in Figure 4. This approach is the continuous interleaved sampling (CIS) strategy [26], which is used as the default strategy or as a processing option in all implant systems now in widespread clinical use.

The CIS strategy filters speech or other input sounds into bands of frequencies with a bank of bandpass filters. Envelope variations in the different bands are represented at corresponding electrodes in the cochlea with modulated trains of biphasic electrical pulses. The envelope signals extracted from the bandpass filters are compressed with a nonlinear mapping function prior to the modulation in order to map the wide dynamic range of sound in the environment (up to about $100 \mathrm{~dB}$ ) into the narrow dynamic range of electrically evoked hearing (about $10 \mathrm{~dB}$ or somewhat higher). The output of each bandpass channel is directed to a single electrode, with low-to-high channels assigned to apical-to-basal electrodes, to mimic at least the order, if not the precise locations, of frequency mapping in the normal cochlea. The

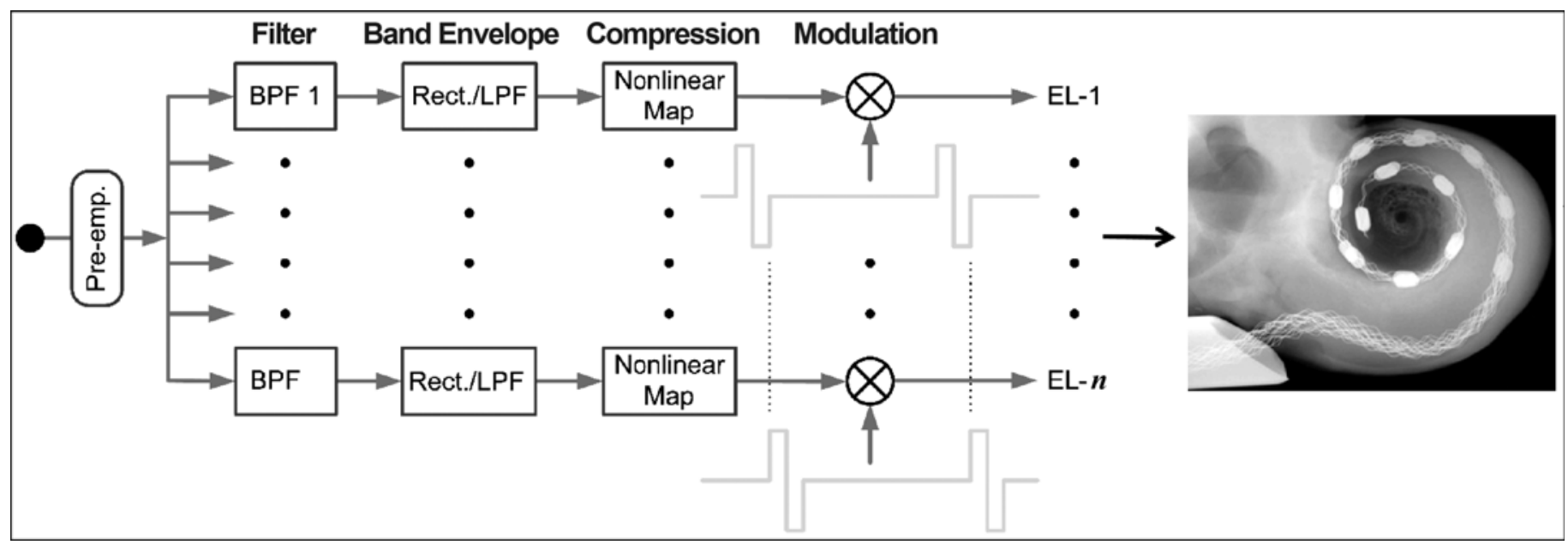

Figure 4.

Continuous interleaved sampling strategy. Input is indicated by filled circle in left-most part of diagram. This input can be provided by microphone or alternative sources such as frequency modulation wireless link in classroom. Following input, strategy uses pre-emphasis filter (Pre-emp.) to attenuate strong components in speech below $1.2 \mathrm{kHz}$. This filter is followed by multiple channels of processing. Each channel includes stages of bandpass filtering (BPF), envelope detection, compression, and modulation. Envelope detectors generally use full-wave or half-wave rectifier (Rect.) followed by low-pass filter (LPF). Hilbert transform or half-wave Rect. without LPF may also be used. Carrier waveforms for two modulators are shown immediately below two corresponding multiplier blocks (circles with “ $x$ ” mark). Outputs of multipliers are directed to intracochlear electrodes (EL-1 to EL- $n$ ) via transcutaneous link (or percutaneous connector in some earlier systems). Inset shows X-ray micrograph of implanted cochlea, to which outputs of speech processor are directed. Source: Block diagram adapted with permission from Wilson BS, Finley CC, Lawson DT, Wolford RD, Eddington DK, Rabinowitz WM. Better speech recognition with cochlear implants. Nature. 1991;352(6332):236-38. [PMID: 1857418]. Inset reprinted with permission from Hüttenbrink KB, Zahnert T, Jolly C, Hofmann G. Movements of cochlear implant electrodes inside the cochlea during insertion: An X-ray microscopy study. Otol Neurotol. 2002;23(2):187-91. [PMID: 11875348] 
pulse trains for the different channels and corresponding electrodes are interleaved in time so that the pulses across channels and electrodes are nonsimultaneous. This eliminates a principal component of electrode interaction, which otherwise would be produced by direct vector summation of the electric fields from different (simultaneously stimulated) electrodes. The corner or "cutoff" frequency of the low-pass filter in each envelope detector is typically set at $200 \mathrm{~Hz}$ or higher so that the fundamental frequencies (F0s) of speech sounds, e.g., $120 \mathrm{~Hz}$ for male voices, are represented (exclusively) in the modulation waveforms. CIS gets its name from the continuous sampling of the (compressed) envelope signals by rapidly presented pulses that are interleaved across electrodes. Between 4 and 22 channels (and corresponding stimulus sites) have been used in CIS implementations to date. (CIS processors are often described as having a small number of channels and associated sites of stimulation, e.g., six to eight, but this is incorrect. The strategy itself does not place a limitation on the number of channels and sites; as just mentioned, CIS implementations to date have used as many as 22 channels and sites.)

Other strategies have also produced outstanding results. Among these are the $n$-of- $m$ [27], spectral peak (SPEAK) [28], advanced combination encoder (ACE) [29], and HiResolution ${ }^{\circledR}$ (HiRes) [30] strategies. The $n$-of- $m$, SPEAK, and ACE strategies each use a channel-selection scheme in which the envelope signals for the different channels are scanned prior to each frame of stimulation across the intracochlear electrodes to identify the signals with the $n$-highest amplitudes from among $m$ processing channels (and associated electrodes). Stimulus pulses are delivered only to the electrodes that correspond to the channels with those highest amplitudes. The parameter $n$ is fixed in the $n$-of- $m$ and ACE strategies and it can vary from frame to frame in the SPEAK strategy, depending on the level and spectral composition of the input signal from the microphone. Stimulus rates typically approximate or exceed 1,000 pulses/s/selected electrode in the $n$-of- $m$ and ACE strategies and approximate 250 pulses/s/selected electrode in the SPEAK strategy. The designs of the $n$-of- $m$ and ACE strategies are essentially identical and are quite similar to CIS except for the channel-selection feature [31]. The SPEAK strategy uses much lower rates of stimulation and an adaptive $n$, as noted previously.

The channel selection or "spectral peak picking" scheme used in the $n$-of- $m$, ACE, and SPEAK strategies is designed in part to reduce the density of stimulation while still representing the most important aspects of the acoustic environment. The deletion of low-amplitude channels (and associated stimuli) for each frame of stimulation may reduce the overall level of masking or interference across electrode and stimulus regions in the cochlea. To the extent that the omitted channels do not contain significant information, such "unmasking” may improve the perception of the input signal by the patient. In addition, for positive speech-to-noise ratios (S/Ns), selection of the channels with the greatest amplitudes in each frame may emphasize the primary speech signal with respect to the noise.

The HiRes strategy is a close variation of CIS that uses relatively high rates of stimulation, relatively high cutoff frequencies for the envelope detectors, and up to 16 processing channels and associated stimulus sites. The terms HiRes and CIS are sometimes used interchangeably. Detailed descriptions of the CIS, $n$-of- $m$, SPEAK, ACE, and HiRes strategies, along with detailed descriptions of many of their predecessors, are presented elsewhere [31].

During the past several years, increasing attention has been paid to representing "fine structure" (FS) or "fine frequency" information with cochlear implants [32-36]. The mathematician David Hilbert showed in 1912 that signals can be decomposed into slowly varying envelopes that modulate high-frequency carriers [37]. An example of such a decomposition is presented in Figure 5. The instantaneous phase, or frequency (the first derivative of the phase signal), of the carrier varies continuously. Hilbert described the carrier as the FS portion of the original signal.

More recently, Zachary Smith and coworkers at the Massachusetts Institute of Technology in Boston, Massachusetts, have investigated the relative importance of envelope and FS information for speech reception, melody reception, and sound lateralization [38]. They created "auditory chimeras" by first processing two separate inputs with identical banks of bandpass filters and then multiplying the FS carriers derived from one bank of filters with the envelope signals derived from the other bank of filters. The modulated carriers were then summed to form the output. Thus, the chimeras presented conflicting cues-the envelope variations in a given number of bands for one sound versus the FS variations in the same bands for another sound. Pairings of inputs included sentences versus noise, sentences versus different sentences, melodies versus different melodies, and 


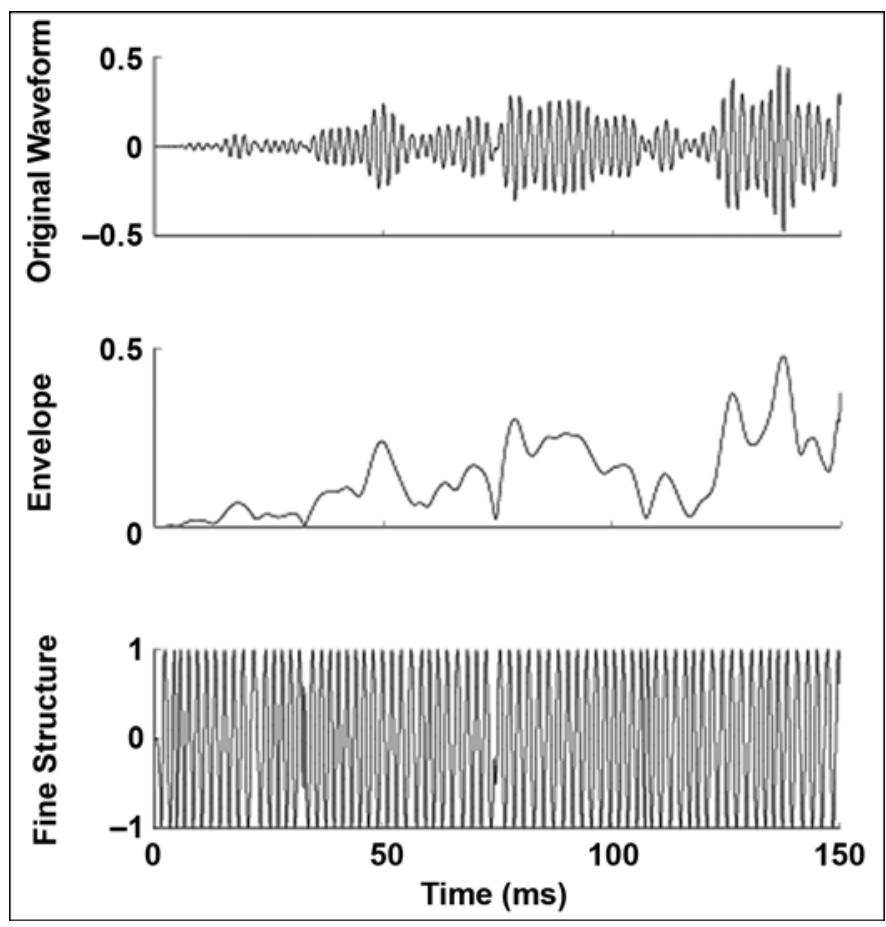

Figure 5.

Decomposition of signal using Hilbert transformation. Illustration is from Smith et al.'s Web site (http://research.meei.harvard.edu/chimera/) and used here with permission of Massachusetts Eye \& Ear Infirmary, Boston, Massachusetts (also see Smith ZM, Delgutte B, Oxenham AJ. Chimaeric sounds reveal dichotomies in auditory perception. Nature. 2002;416(6876):87-90. [PMID: 11882898]).

sentences with an interaural time delay (ITD) corresponding to a sound image at the left side of a subject versus the same sentence or different sentences with an ITD corresponding to a sound image at the right side of a subject.

The sound heard or correctly identified by subjects with normal hearing depended on the type(s) of sounds in each pairing and on the number of processing channels (bands). Speech was identified by its envelope information for eight or more channels, whereas the FS information was more important for one or two channels. Both envelope and FS information contributed to sentence recognition for intermediate numbers of channels. Melodies were recognized almost exclusively by their FS information for up to 32 channels. Envelope cues became dominant at 48 and 64 channels. Lateralization of sentences was difficult for the subjects with a small number of channels, but performance improved with increasing numbers up to the tested limit of 32. Lateralization was cued by the FS information in all cases.
These findings indicate the importance of FS information for speech reception using fewer than about 8 processing channels and for music reception using fewer than about 40 channels. In addition, the findings indicate that ITD cues may be represented by FS information but not envelope information for any number of channels up to (at least) 32.

As mentioned previously, present-day electrode arrays for cochlear implants do not support more than four to eight channels of received or perceptually separable information. In this four to eight range, both envelope and FS information contribute to speech reception. Music information is conveyed almost solely by FS cues.

In the processing strategies described thus far, envelope signals are derived from the outputs of bandpass filters and those signals are used to determine the patterns of stimulation at the electrode array. However, the division between the envelope and FS parts of the input is not as clearly delineated in these strategies for implants as in the study by Smith et al. [38]. Although only envelope information is presented with the strategies, frequencies included in the envelopes generally range up to 200 to $400 \mathrm{~Hz}$ or even higher (in the HiRes strategy). Thus, substantial FS information is presented and may be at least partially perceived in this low-frequency range. In addition, and as described later in this article, frequencies between the center frequencies of adjacent bandpass channels can be conveyed with cochlear implants by adjusting the ratio of stimulus pulse amplitudes for the corresponding electrodes. A finely graded representation of frequencies may be achieved in this way with implants but not with the envelope part of the auditory chimeras studied by Smith et al. In particular, the envelope-based strategies for implants may transmit at least some FS or "fine frequency" information via simultaneous or rapid sequential stimulation of adjacent electrodes and a resulting "channel balance" cue to intermediate frequencies that excite both of the corresponding bandpass channels. (Excitation of adjacent channels with a single frequency component results from the overlapping frequency responses of the bandpass filters for the channels.)

At this time, how much FS information is presented and received with the envelope-based strategies is not clear. The possibility that only a small amount of the information is transmitted, along with the findings of Smith et al. [38] demonstrating the importance of the information, have motivated multiple efforts (e.g., [3233]) to represent the information in other ways. Indeed, 
many have assumed that little or no FS information is transmitted by the envelope-based strategies, because "only envelope information is presented." This assumption ignores the fact, however, that temporal information is presented in the modulation waveforms up to 200 to $400 \mathrm{~Hz}$ or higher and the fact that a channel-balance cue may well convey at least some FS information at higher frequencies.

A difficulty shared by the present and proposed approaches is that changes in the rate of stimulation for a given electrode or collection of electrodes are not perceived as differences in pitch above a "pitch saturation limit" of about $300 \mathrm{~Hz}$ for most patients [39]. (Some patients have higher limits, up to about $1,000 \mathrm{~Hz}$ or somewhat higher, but these patients are the rare exceptions to the general rule.) In addition, the difference limens for frequency changes below $300 \mathrm{~Hz}$ are generally much worse (typically 10 times worse) for implant patients than for listeners with normal hearing [39-40]. Thus, representation of FS information by a temporal code- such as the timing or frequency of pulse presentations within channels-may be limited to $300 \mathrm{~Hz}$ or lower and may be highly degraded compared with normal, even within that restricted low-frequency range.

A further difficulty is that the effective number of sites of stimulation along the length of the electrode array appears to be limited to about four to eight for present placements and designs of the arrays, even for arrays with many more electrodes than that and even though more sites can be discriminated on the basis of pitch by many patients. (The apparent discrepancy between the number of effective channels in a speech or multichannel context versus the number of sites that can be discriminated when stimulated in isolation remains as a mystery.) Thus, representation of FS (or the fine frequency) information with a fine-grained adjustment in the site or sites of stimulation may be highly limited as well, even with the channel-balance cue described previously.

Despite these difficulties and likely limitations, new processing options have been introduced by two of the three principal manufacturers of implant systems that are designed to increase the transmission of FS information compared with the CIS and other strategies in current widespread use. In one approach, the timing of positive zero crossings in the output of the bandpass filter with the lowest center frequency, or in the outputs of up to four bandpass filters with the lowest center frequencies, is "marked" with the presentation of a short group of pulses for the corresponding channel(s) and site(s) of stimulation as opposed to the continuous presentation of pulses for standard CIS channels. The overall amplitude of the pulse bursts for these special processing channels is determined by the magnitude of energy in the band for each channel, as in CIS. The remaining higher frequency channels use CIS processing and present pulses continuously, interlaced across electrodes. The pulses for the lower frequency channels are also interlaced across electrodes, including the electrodes presenting the CIS stimuli. (This requirement of nonsimultaneity can degrade the precision of marking the zero crossings by pulse presentations, especially when long pulse durations must be used and especially for a high number of activated electrodes in the implant.)

This strategy is called the "fine structure processing" (FSP) strategy [35-36] and is similar in design to a strategy described by Wilson et al. in 1991 [41], called the "peak picker/CIS" (PP/CIS) strategy. The principal difference between the FSP and PP/CIS strategies is that single pulses are presented at peaks in the bandpass filter outputs in the PP/CIS strategy, whereas groups of pulses (including the possibility of a single pulse) are presented at the zero crossings in the FSP strategy.

The FSP and related approaches may provide an advantage compared with CIS and other envelope-based strategies to the extent that single pulses or short groups of pulses represent temporal events in the lower channel(s) better than the continuous (and time varying) modulations for the same channels in envelope-based strategies. Some evidence exists for this postulated advantage [35-36,4142], and studies are in progress to evaluate further the possible benefits of the FSP approach for speech or music reception.

The other approach noted previously is to represent the fine frequency information within bands using multiple sites of stimulation for each band and associated channel rather than the single site for each band and channel used in CIS and other strategies. This approach is a variation of HiRes (and CIS) and is called the HiRes with the Fidelity $120^{\mathrm{TM}}$ option (HiRes 120). It employs "virtual channels" as a way to increase the number of discriminable sites beyond the number of physical electrodes. This concept of virtual channels for CIS-like processors was introduced by Wilson et al. in the early 1990s [43-46] and has since been investigated by others [47-51]. In some of these reports, the term "current steering" is used instead of the term virtual channels to reference the same concept. 
A series of diagrams illustrating the construction of virtual channels is presented in Figure 6. With virtual channels (or current steering), adjacent electrodes may be stimulated simultaneously to shift the perceived pitch in any direction with respect to the percepts elicited with stimulation of either of the electrodes alone. Results from studies with implant subjects indicate that pitch can be manipulated through various choices of simultaneous and single-electrode conditions (e.g., Wilson et al. [44]). If, for instance, the apical-most electrode in an array of electrodes is stimulated alone (electrode 1, Figure 6(a)), subjects have reported a low pitch. If the next electrode in the array is stimulated alone (electrode 2, Figure 6(b)), a higher pitch is reported. An intermediate pitch can be produced for the great majority of subjects studied to date by stimulating the two electrodes together with identical in-phase pulses (Figure 6(c)). Finally, the pitch elicited by stimulation of a single electrode can also be shifted by presentation of an opposite-polarity pulse to a neighboring electrode. For example, a pitch lower than that elicited by stimulation of electrode 1 alone can be produced by simultaneous presentation of a (generally smaller) pulse of opposite polarity at electrode 2 (Figure 6(d)). The availability of pitches other than those elicited with stimulation of single electrodes alone may provide additional discriminable sites along (and beyond) the length of the electrode array. Such additional sites may (or may not) support a higher number of effective information channels with implants compared with stimulation that is restricted to single electrodes only.

The concept of virtual channels can be extended to include a quite high number of sites and corresponding pitches by using different ratios of the currents delivered between simultaneously stimulated electrodes. This possibility is illustrated in Figure 7, in which stimulus site 1 is produced by stimulation of electrode 1 alone, stimulus site 2 by simultaneous stimulation of electrodes 1 and 2 with a pulse amplitude of 75 percent for electrode 1 and 25 percent for electrode 2 , and so on. The total number of sites and corresponding pitches that might be produced for a good subject in the illustrated case is 21, with 6 intracochlear electrodes. (A subject was tested with this arrangement and indeed obtained 21 discriminable pitches [52].) Other ratios of currents may produce additional pitches. Results from several recent studies have indicated that a high number of discriminable pitches can be created with this general approach, e.g., Koch et al. [51] found an average of 93 (range 8-466) discriminable pitches for a

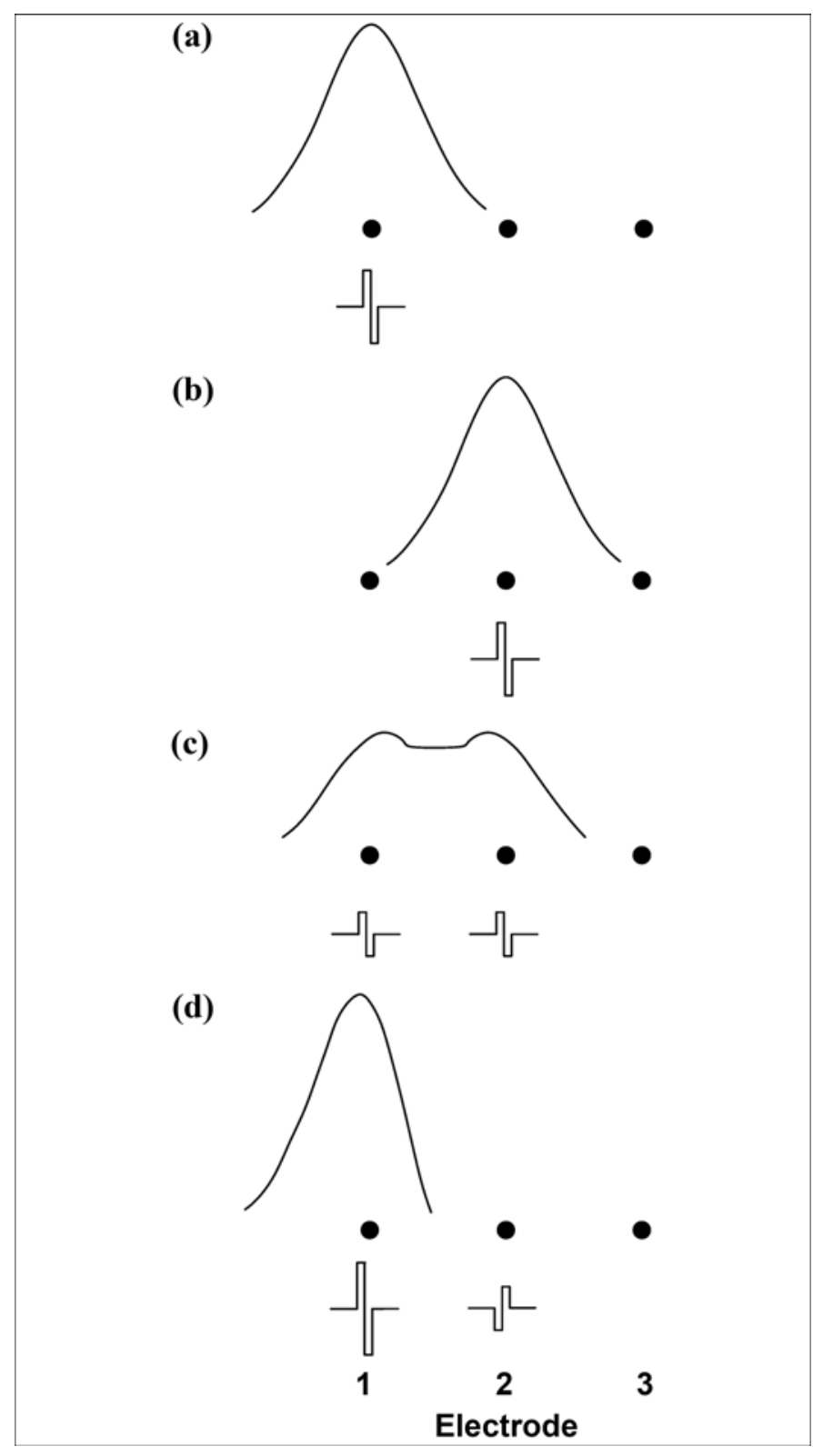

Figure 6.

Schematic illustration of neural responses for various conditions of stimulation with (a)-(b) single and (c)-(d) multiple electrodes. Top curve in each panel is hypothetical sketch of number of neural responses, as function of position along length of cochlea for given condition of stimulation. Condition is indicated by pulse waveform(s) beneath one or more dots, which represent positions of three adjacent intracochlear electrodes. These different conditions of stimulation elicit distinct pitches for implant patients; see main text for full discussion. Source: Reprinted with permission from Wilson BS, Schatzer R, Lopez-Poveda EA. Possibilities for a closer mimicking of normal auditory functions with cochlear implants. In: Waltzman SB, Roland JT Jr, editors. Cochlear implants. 2nd ed. New York (NY): Thieme; 2006. p. 48-56. 


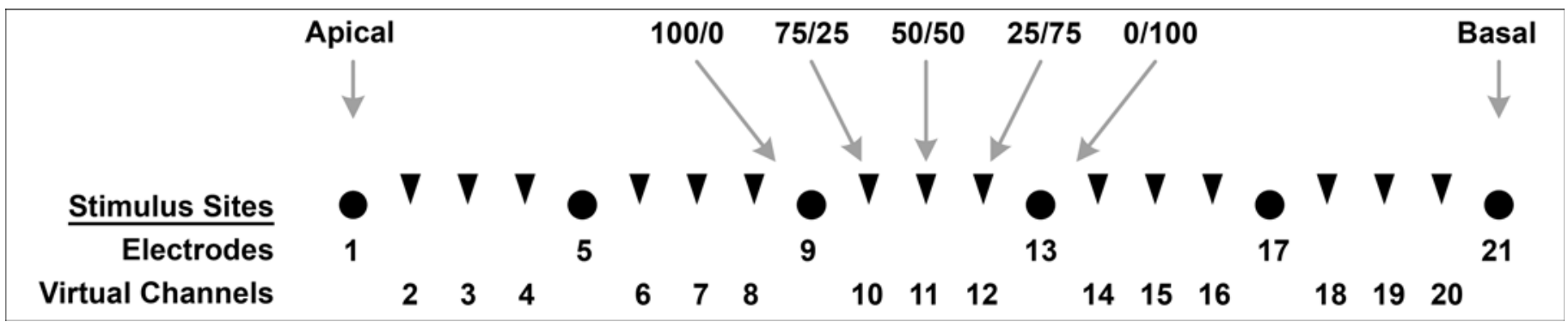

Figure 7.

Diagram of stimulus sites used in virtual channel interleaved sampling processors and other similar processors that followed them. Filled circles represent sites of stimulation at each of 6 intracochlear electrodes. Inverted triangles represent additional sites produced with simultaneous stimulation of adjacent electrodes at indicated ratios of pulse amplitudes for 2 electrodes. Thus, in this arrangement, 21 sites may be produced, including 6 electrodes and 15 "virtual” sites, between simultaneously stimulated electrodes. More electrodes may be used, and more sites may be formed between adjacent electrodes, e.g., as in 120 sites produced with HiResolution with Fidelity 120 option strategy. Some patients are able to discriminate high number of sites on basis of pitch; see main text for full discussion. Source: Reprinted with permission from Wilson BS, Schatzer R, Lopez-Poveda EA. Possibilities for a closer mimicking of normal auditory functions with cochlear implants. In: Waltzman SB, Roland JT Jr, editors. Cochlear implants. 2nd ed. New York (NY): Thieme; 2006. p. 48-56.

large population of subjects using either of two versions of the Advanced Bionics Corp (Valencia, California) electrode array, both of which included 16 physical intracochlear electrodes spaced $\sim 1 \mathrm{~mm}$ apart. (A very few subjects did not perceive pitch differences even with stimulation of adjacent or more distant electrodes in isolation, producing a number of discriminable pitches that was less than the number of physical electrodes.)

In the HiRes 120 strategy, 8 sites are allocated to each of 15 bandpass ranges to form 120 sites in all. The different sites for each channel are produced with eight different ratios of currents delivered to the two adjacent electrodes assigned to that bandpass range. One of the eight ratios is used at the time of stimulation for each of the channels, and the stimuli for the different channels are presented in a nonoverlapping sequence, just as in the CIS strategy. Unlike the CIS strategy, however, two electrodes are stimulated together (with the selected amplitude ratio) at each update, rather than stimulation of a single electrode at each update. The ratio for each bandpass range and update in the HiRes 120 strategy is selected to correspond to the frequency of the strongest component within the range at that time. More specifically, eight "subbands" within the range are sampled just prior to each stimulus update and the current ratio for the two electrodes is selected to correspond to the subband with the greatest energy.

The idea underlying the HiRes 120 strategy and the "virtual channel interleaved sampling” (VCIS) strategy that preceded it [46] is that a high number of available pitches may allow patients access to relatively small frequency differences of components in speech, music, and other sounds. As noted previously, such access might be very helpful for speech reception, particularly speech reception in adverse conditions, and such access may be essential for music reception, which is generally quite poor with the CIS and other related strategies, as might be expected from the findings of Smith et al. [38] reviewed previously and assuming that only a modest amount of FS information is transmitted by those strategies.

Several studies are underway to evaluate the possibility of a speech reception or music reception advantage with HiRes 120. We expect that complete data from those studies will be available soon.

We note that (1) a high number of available pitches or discriminable sites does not guarantee a high number of effective channels with cochlear implants, as previously mentioned, and (2) "virtual pitches" may well be inherent in standard CIS and related strategies using sequential stimulation, in that intermediate pitches also can be produced with nonsimultaneous stimulation of adjacent (or more distant) electrodes so long as the pulses are relatively close in time [53-55]. Thus, strategies that explicitly code virtual channels through simultaneous stimulation of adjacent electrodes may not increase the number of effective channels, or even the number of available pitches, compared with the CIS and related strategies. In this regard, we also note that VCIS processors, which were evaluated 
in the early 1990s using measures of speech reception in quiet, were not statistically better for any of the measures than the control CIS processors. These early studies did not include measures of music reception or of speech reception in competition with noise or other talkers; nor did they include a large number of subjects. Possibly, results from other tests or the higher statistical power afforded by a larger number of subjects will demonstrate differences between HiRes and HiRes 120. We will have to wait for the data to know whether any differences exist and, if so, for what measures.

Applications of the processing strategies in current use according to manufacturer are shown in the Table. The three major manufacturers of implant devices are listed and include MED-EL Medical Electronics GmbH of Innsbruck, Austria; Cochlear Ltd of Lane Cove, Australia; and Advanced Bionics Corp. CIS is the default strategy for the MED-EL device, HiRes is the default choice for the Advanced Bionics device, and ACE is the default strategy for the Cochlear device. Optional or alternative strategies are offered by each of the manufacturers as also indicated in the Table. An alternative strategy may be selected by the audiologist at the time of a first or subsequent fitting for a particular patient. However, alternate strategies are rarely applied, and the default choices are generally the ones used in standard clinical practice, at least as of this writing (September 2007).

\section{PERFORMANCE WITH PRESENT-DAY SYSTEMS}

\section{Average Performance and Range of Scores}

Each of these highly utilized strategies-CIS, HiRes, and ACE-supports recognition of monosyllabic words on the order of 50 percent correct (using hearing alone) across populations of tested subjects (see Table 2.4 in
Wilson [31]). Variability in outcomes is high, however, with some subjects achieving scores at or near 100 percent correct and other subjects scoring close to zero on this most difficult of standard audiological measures. Standard deviation values of the scores range from about 10 percent to about 30 percent for the various studies conducted to date. Scores for the monosyllabic word tests are not significantly different from each other among the three strategies, nor are the scores for a wide range of other speech reception measures.

However, differences in performance can be found for groups of patients using different implant systems if the tests are made more difficult than those used in clinical practice. For example, if patients are tested in noise and at soft presentation levels, then systems with a large input dynamic range outperform systems with a small input dynamic range [56]. This outcome emphasizes that the easy-to-see differences among systems, such as number of processing channels, pulse rate, and processing strategy, are not always the differences that make a difference in performance. The details of the hardware implementation of common components, e.g., the input dynamic range or the shape of the compression function, can be critical when performance is tested across difficult listening conditions. (A further discussion about the importance of hardware-and software-implementations is presented in Wilson [22], especially in section 4.3, "Strategy Implementations.")

The ranges of scores and other representative findings for contemporary cochlear implants are illustrated in Figure 8, which shows scores for 55 users of the MEDEL COMBI 40 implant system with the CIS processing strategy. Scores for the Hochmair-Schultz-Moser sentences are presented in Figure 8(a), and scores for recognition of the Freiburger monosyllabic words are

Table.

Processing strategies in current use for cochlear implants. Manufacturers are shown in left column and processing strategies used in their implant systems are shown in remaining columns.

\begin{tabular}{lccccccc}
\hline \multicolumn{1}{c}{ Manufacturer } & CIS & n-of-m & ACE & SPEAK & FSP & HiRes & HiRes 120 \\
\hline MED-EL Medical Electronics GmbH & $\mathrm{X}$ & $\mathrm{X}$ & - & - & $\mathrm{X}$ & - & - \\
Cochlear Ltd $^{\dagger}$ & $\mathrm{X}$ & - & $\mathrm{X}$ & $\mathrm{X}$ & - & - & - \\
Advanced Bionics Corp $^{\ddagger}$ & $\mathrm{X}$ & - & - & - & - & $\mathrm{X}$ & $\mathrm{X}$ \\
\hline
\end{tabular}

*Innsbruck, Austria.

${ }^{\dagger}$ Lane Cove, Australia.

${ }_{\ddagger}^{\ddagger}$ Valencia, California.

ACE $=$ advanced combination encoder, CIS $=$ continuous interleaved sampling, FSP $=$ fine structure processing, HiRes $=$ HiResolution, HiRes $120=$ HiRes with Fidelity 120 option, SPEAK = spectral peak. 
presented in Figure 8(b). Results for five measurement intervals are shown, ranging from 1 to 24 months following the initial fitting of the speech processor. The solid line in each panel shows the median of the individual scores, and the dashed and dotted lines show the interquartile ranges. The data are a superset of those reported in Helms et al. [57] and include scores for additional subjects at various test intervals.

Most of the subjects used an eight-channel processor with a pulse rate of about 1,500/s/electrode. Some of the subjects used fewer channels and a proportionately higher rate. (All processors used the maximum overall rate of 12,120 pulses/s across electrodes.)

As is evident from the figure, scores are broadly distributed at each test interval and for both tests. However, ceiling effects are encountered for the sentence test for many of the subjects, especially at the later test intervals. At 24 months postfitting, 46 of the 55 subjects score above 80 percent correct, consistent with the conclusion quoted previously from the 1995 NIH Consensus Conference on Cochlear Implants in Adults and Children. Scores for the recognition of monosyllabic words are much more broadly distributed. For example, at the 24-month interval, only 9 of the 55 subjects have scores above 80 percent correct and the distribution of scores from about 10 percent correct to nearly 100 percent correct is almost perfectly uniform.

An interesting aspect of the results presented in Figure 8 is the improvement in performance over time. This improvement is easiest to see in the lower ranges of scores, e.g., in the steady increase in the lower interquartile lines (the dotted lines) across test intervals.

Improvements over time are even more evident in plots of mean scores for sentences and for words, as shown in Figure 9 for these same data and for additional test intervals for the sentence test. The mean scores increase for both the sentence and word tests out to 12 months and then plateau thereafter. The mean scores for the sentence test asymptote at about 90 percent correct, and the mean scores for the word test asymptote at about 55 percent correct. Such results typify performance with the best of the modern cochlear implant systems and processing strategies for electrical stimulation on one side with a unilateral implant.

These results are especially remarkable for the top scorers, given that only a maximum of eight broadly overlapping sectors of the auditory nerve are stimulated with this device and the implementation of CIS used with it.
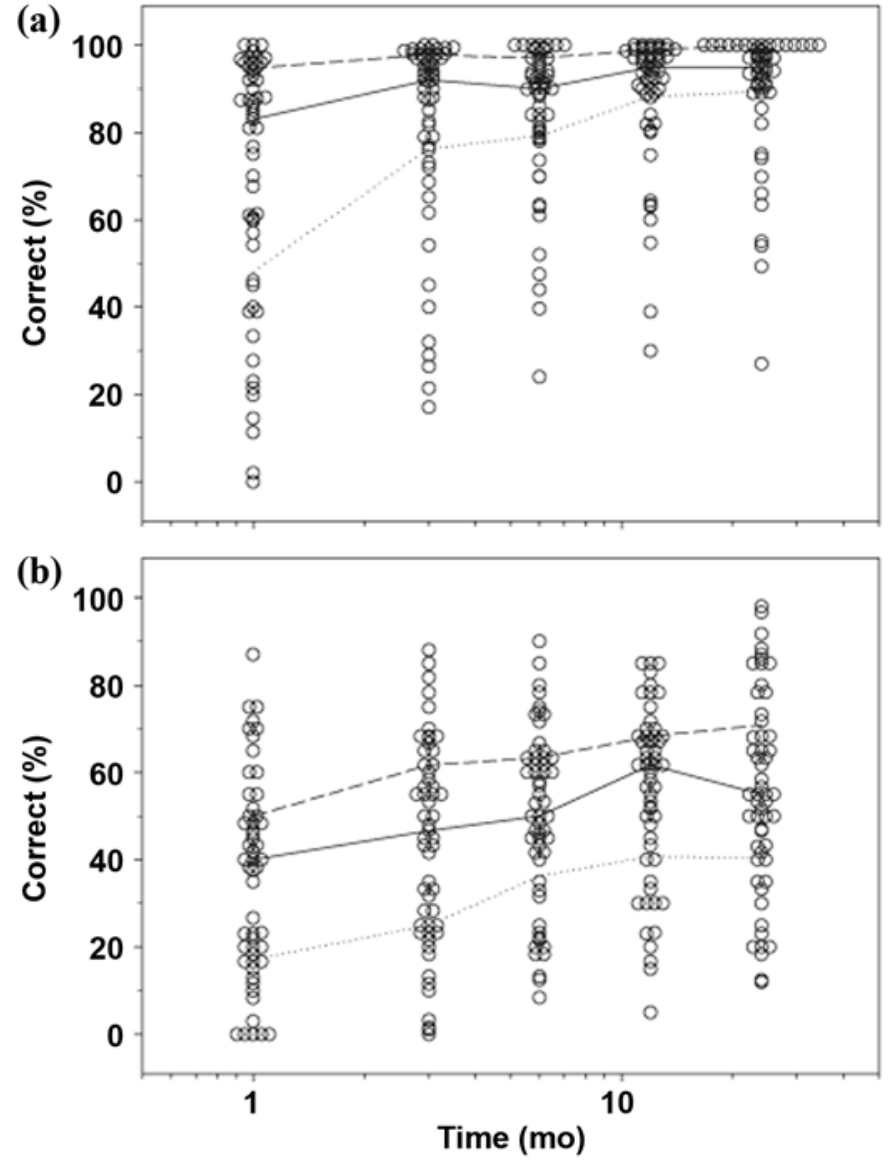

Figure 8.

Percent correct scores for 55 users of COMBI 40 implant (MED-EL Medical Electronics GmbH; Innsbruck, Austria) and CIS processing strategy. Scores for recognition of (a) Hochmair-Schultz-Moser sentences and (b) Freiburger monosyllabic words are presented. Solid line in each panel shows median scores, and dashed and dotted lines show interquartile ranges. Data are updated superset of those reported in Helms J, Müller J, Schön F, Moser L, Arnold W, Janssen T, Ramsden R, Von Ilberg C, Kiefer J, Pfennigdorf T, Gstöttner W, Baumgartner W, Ehrenberger K, Skarzynski H, Ribari O, Thumfart W, Stephan K, Mann W, Heinemann M, Zorowka P, Lippert KL, Zenner HP, Bohndord M, Hüttenbrink K, Hochmair-Desoyer I, et al. Evaluation of performance with the COMBI40 cochlear implant in adults: A multicentric clinical study. ORL J Otorhinolaryngol Relat Spec. 1997; 59(1):23-35. [PMID: 9104746], kindly provided by Patrick D’Haese of MED-EL GmbH. Experimental conditions and implantation criteria are also described in Helms et al. All subjects took both tests at each of indicated intervals following initial fitting of their speech processors. Identical scores at single test interval are displaced horizontally for clarity. Thus, for example, horizontal "line" of scores in top right portion of (a) represent scores for 24-month test interval. Source: Reprinted with permission from Wilson BS. Speech processing strategies. In: Cooper H, Craddock LC, editors. Cochlear implants: A practical guide. 2nd ed. London (England): Whurr; 2006. p. 21-69. 
This number is quite small in comparison to the normal complement of approximately 30,000 neurons in the human auditory nerve.

The results also show a learning or accommodation effect, with continuous improvements in scores over the first 12 months of use. This demonstrated effect for implants suggests the likely importance of brain function in determining outcomes and the reorganization (brain plasticity) that must occur for patients to use such sparse inputs to the maximum extent possible.

\section{Top Performers}

The top performers with present-day cochlear implants can achieve remarkably high scores in tests of speech recognition. Scores for one such subject, implant subject HR4, are shown in the black bars in Figure $\mathbf{1 0}$ for a comprehensive set of tests. Mean scores for six undergraduate students with normal hearing for the same tests are shown in the gray bars, along with the standard error of the mean for each test. Subject HR4 was totally deaf prior to receiving his implant. The tests included (1) recognition of monosyllabic consonant-nucleus-consonant (CNC) words (50 items), (2) recognition of City University of New York (CUNY) sentences (24 sentences and

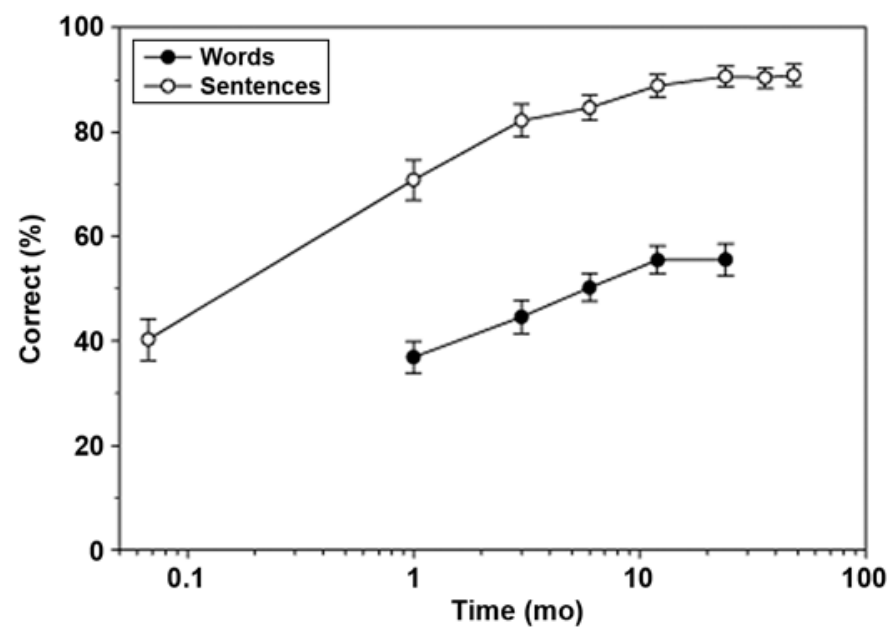

Figure 9.

Mean and standard error of the mean for 54 of 55 subjects in Figure 8. (One subject did not take sentence test for expanded range of intervals so these data are not presented here.) Additional interval before and two intervals after those indicated in Figure 8 were used for sentence test. Source: Reprinted with permission from Wilson BS. Speech processing strategies. In: Cooper H, Craddock LC, editors. Cochlear implants: A practical guide. 2nd ed. London (England): Whurr; 2006. p. 21-69. approximately 200 words, depending on the lists used for each subject), (3) recognition of Hearing in Noise Test sentences (250 sentences and 1,320 words, presented in quiet), (4) recognition of the Arizona Biomedical (AzBio) sentences (40 sentences and approximately 270 words, depending on the lists used), (5) identification of 20 consonants in an /e/-consonant-/e/ context (with 5 repetitions of the 20 in randomized order), (6) identification of 13 computer-synthesized vowels in a /b/-vowel-/t/ context (with 5 repetitions of the 13 in randomized order), and (7) recognition of CUNY and AzBio sentences presented in competition with a four-talker babble at the speech-tobabble ratio $(\mathrm{S} / \mathrm{B})$ of $+10 \mathrm{~dB}$ for the CUNY sentences and at that ratio and at $+5 \mathrm{~dB}$ for the AzBio sentences. Further details about the subjects, tests, and testing procedures are presented in Wilson and Dorman [58].

Figure 10 shows a spectacular restoration of function for a user of a sensory neural prosthesis. All of the scores

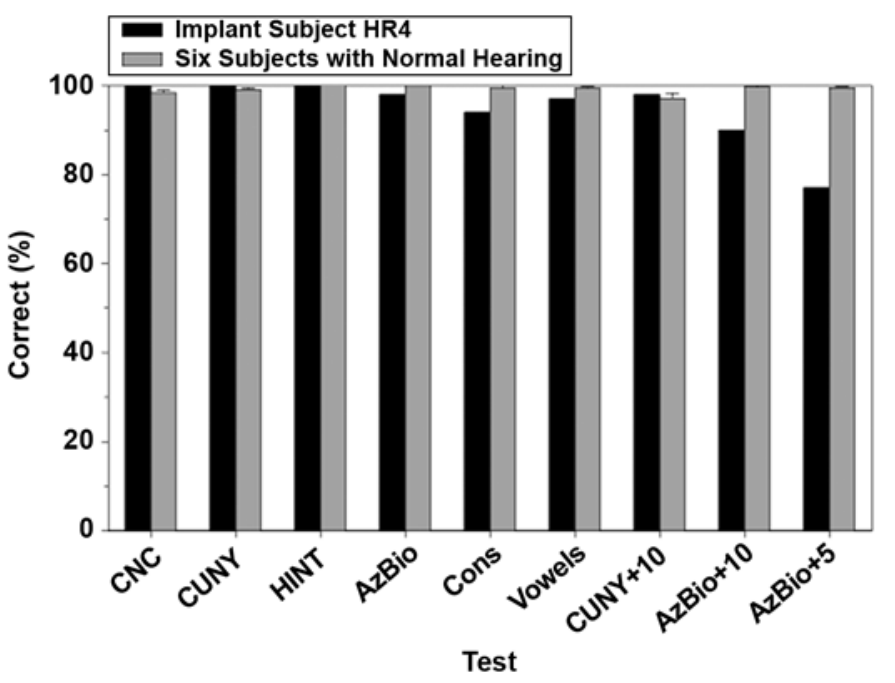

Figure 10.

Percent-correct scores for implant subject HR4 and for six subjects with normal hearing. Means and standard error of the means are shown for subjects with normal hearing. Tests included recognition of monosyllabic consonant-nucleus-consonant (CNC) words; recognition of City University of New York (CUNY) sentences; recognition of Hearing in Noise Test (HINT) sentences; recognition of Arizona Biomedical (AzBio) sentences; identification of consonants (Cons) in /e/-consonant-/e/ context; identification of vowels in /b/vowel-/t/ context; and recognition of CUNY and AzBio sentences presented in competition with four-talker babble, at indicated speechto-babble ratios $(+5$ or $+10 \mathrm{~dB})$. Source: Reprinted with permission from Wilson BS, Dorman MF. The surprising performance of present-day cochlear implants. IEEE Trans Biomed Eng. 2007;54 (6 Pt 1):969-72. [PMID: 17554816]. (C) 2007 IEEE. 
for subject HR4 are high. His scores for speech material presented in quiet, including words, sentences, consonants, and vowels, match or closely approximate the scores for the control group. His score for the most difficult test used in standard clinical practice, recognition of the monosyllabic CNC words, is 100 percent correct. In contrast, some of his scores for sentences presented in competition with speech babble are worse than normal. Although his score for the CUNY sentences at the S/B of $+10 \mathrm{~dB}$ is 98 percent correct, his scores for the AzBio sentences at the $\mathrm{S} / \mathrm{Bs}$ of $+10 \mathrm{~dB}$ and $+5 \mathrm{~dB}$ are below those of the normal-hearing subjects. In all, subject HR4 scored at or near the ceiling of 100 percent correct for seven of the nine tests and he attained scores of 77 percent correct or better for the remaining two tests. (The subjects with normal hearing scored at or near the ceiling for all nine tests.) Subject HR4 scored at the ceiling for all tests given in standard clinical practice to identify deficits in hearing. His results indicate a full restoration of clinically normal function, at least for speech reception. He used a 16-channel CIS processor, as implemented in the Clarion ${ }^{\circledR}$ CII cochlear prosthesis (Advanced Bionics Corp) [59]. This prosthesis also includes a high-bandwidth transcutaneous link, current sources with short rise and fall times, an array of 16 intracochlear electrodes, and (in the version used) a positioning device to place the electrodes next to the inner wall of the ST.

Such high scores overall are consistent with subject HR4's ability to communicate with ease in most listening situations. He has no difficulty at all in telephone communications. He can understand conversations not directed to him and can identify speakers by regional dialect. He can mimic voices and accents that he has heard only after receiving the implant. His speech reception abilities are truly remarkable, abilities that could not have been imagined 20 years ago, even by the most optimistic proponents of cochlear implants.

Other patients using this and other implant systems, and also other processing strategies (including the $n$-of- $m$ and ACE strategies), have achieved high scores as well. For example, one of the subjects in Figure 8 achieved a score of 98 percent correct in the Freiburger monosyllabic word test at the 2-year interval. This subject used a COMBI 40 implant system, with its eight channels of CIS processing and eight sites of stimulation. This system also has a high-bandwidth transcutaneous link and current sources with short rise and fall times. It does not include a positioning device, nor do other versions of the
Clarion prosthesis or other implant systems that also support stellar scores for some patients.

Although more than a few patients have achieved scores like those shown in Figure 10, most patients have lower scores, typically much lower scores for the difficult tests, as also indicated in Figure 8(b). However, the results obtained from studies with subject HR4 and his peers are proof of what is possible with electrical stimulation of the auditory nerve in a totally deafened ear.

\section{STRENGTHS AND LIMITATIONS OF PRESENT- DAY SYSTEMS}

\section{Efficacy of Sparse Representations}

Some patients achieve spectacularly high scores with present-day cochlear implants. Indeed, their scores are in the normal ranges, even for the most difficult of standard audiological tests. Such results are both encouraging and surprising in that the implants provide only a very crude mimicking of only some aspects of the normal physiology. In cases like that of subject HR4, 16 overlapping sectors of the auditory nerve are stimulated with 16 intracochlear electrodes. As noted previously, in other cases, other patients have achieved similarly high scores with eight sites of stimulation in the cochlea. (High scores, e.g., in the low-to-mid 90s for recognition of monosyllabic words, can on rare occasions be obtained even with as few as six channels of CIS processing and stimulation $[46,60]$.) The spatial specificity of stimulation with implants is much lower than that demonstrated in neural tuning curves for normal hearing [61], especially for monopolar stimulation, which is used in all present-day systems. As also noted previously, such broad and highly overlapping activation of the nerve most likely limits the number of perceptually separable channels to four to eight, even if more than eight electrodes are used. The information presented through the implant is limited to envelope variations in the 16 or fewer frequency bands for these patients. (Similar numbers apply for patients achieving high scores with processing strategies other than CIS.) For subject HR4 and others, the upper frequency of envelope variations has been set at 200 to $700 \mathrm{~Hz}$ [31], e.g., by using a cutoff frequency in the range of 200 to $700 \mathrm{~Hz}$ for the low-pass filters in the envelope detectors shown in Figure 4. A substantial fraction of this information may be perceived by the better 
patients [60,62-63] and whatever is perceived is sufficient for high levels of speech recognition.

The performance achieved by subject HR4 and others like him brings into question the significance for speech reception of the intricate processing and the interplay between and among processing steps that occur in the normal cochlea. The details of the traveling wave of mechanical displacements along the BM in response to acoustic stimuli [64] and the spatial sharpening of the membrane response by active processes at the OHCs [64-65] are not necessary for effective representations of speech information. Also, the noninstantaneous compression function at the synapses between the IHCs and single fibers of the auditory nerve [66] is not necessary. Additional aspects of normal hearing that are not replicated with implants include multiple stages of compression (at the BM/OHC complex, at the IHCs, and at the IHC/neuron synapses); effects of efferent action on the OHCs and other structures in the cochlea [67-68]; the broad distributions of thresholds for the multiple afferent fibers innervating each IHC [69]; and effects of spontaneous activity in the nerve [70], which is absent or nearly so in the deafened ear [71-73]. Despite these many missing steps or severed connections, cochlear implants can restore clinically normal function in terms of speech reception for some patients, which is remarkable.

\section{Possible Deficit in Representing Fine Structure Information}

At present, we do not know how much FS information is transmitted with the "envelope-based" strategies such as CIS, HiRes, and ACE. The amount may be small. As noted previously, FS information may be important for speech reception under adverse conditions, and it may well be essential for music reception beyond perception of gross aspects such as rhythm or small sets of simple melodies. The FSP and HiRes 120 strategies are designed to improve the representation of FS information. However, limitations in perception with implants may impose "roadblocks" to the success of either or both of these strategies. The same limitations may apply to other approaches that have been proposed for a better representation of FS information. Presenting the information in a way that it can be perceived and utilized by patients is a difficult and active area of research. We should know more about the performances of the FSP and HiRes 120 strategies, and possibly one or more of the other approaches, in the near future.

\section{Variability in Outcomes}

One of the major remaining problems with cochlear implants is the broad distribution of outcomes, especially for difficult tests and as exemplified in Figure 8(b). That is, patients using exactly the same implant system-with the same speech processor, transcutaneous link, implanted receiver/stimulator, and implanted electrode array-can have scores ranging from the floor to the ceiling for such tests. Indeed, only a small fraction of patients achieve the spectacularly high scores discussed previously.

\section{Likely Limitations Imposed by Impairments in Auditory Pathway or Cortical Function}

Accumulating and compelling evidence is pointing to differences in cortical or auditory pathway function as a contributor to the variability in outcomes with cochlear implants. On average, patients with short durations of deafness prior to their implants fare better than patients with long durations of deafness [74]. This may be the result of sensory deprivation for long periods, which adversely affects connections between and among neurons in the central auditory system [75] and may allow encroachment by other sensory inputs of cortical areas normally devoted to auditory processing (this encroachment is called "cross-modal plasticity" [76-77]). Although one might think that differences in nerve survival at the periphery could explain the variability, either a negative correlation or no relationship has been found between the number of surviving ganglion cells and prior word recognition scores for deceased implant patients who had agreed to donate their temporal bones (containing the cochlea) for postmortem histological studies [78-81]. In some cases, survival of the ganglion cells was far shy of the normal complement, and yet these same patients achieved high scores on monosyllabic word tests. Conversely, in some other cases, survival of the ganglion cells was excellent, and yet these patients did not achieve high scores on the tests. Although some number of ganglion cells must be required for the function of a cochlear implant, this number appears to be small. Above that putative threshold, the brains of the better-performing patients apparently can use a sparse input from even a small number of surviving cells for high levels of speech reception.

Similarly, the representation of speech sounds with a cochlear implant likely needs to be above some threshold in order for the brain to utilize the input for good speech reception. Single-channel implant systems did not rise above this second putative threshold for all but a few 
exceptional patients, nor did prior processing strategies for multichannel implants. The combination of multiple sites of stimulation in the cochlea (at least six to eight); the current processing strategies such as CIS, HiRes, $n$ of- $m$, and ACE; and some minimum survival of ganglion cells are sufficient for a high restoration of function in a relatively high number of patients. Those patients are likely to have intact or largely intact "auditory brains" that can use these inputs that are still sparse and distorted compared with the inputs received by the brain from the normal cochlea.

Other patients may not have the benefit of normal or nearly normal processing central to the auditory nerve. The effects of auditory deprivation for long periods have been mentioned. In addition, the brains of children become less "plastic" or adaptable to new inputs beyond their third or fourth birthday. This may explain why deaf children implanted before then generally have much better outcomes than deaf children implanted at age 5 and older [76,82-83].

Two examples of recent findings implicating the importance of brain function in determining outcomes with cochlear implants are presented in Figures 11 and 12. Figure 11 presents results from a study conducted by Anu Sharma and coworkers at the University of Texas at Dallas and at Arizona State University [82], and Figure 12 pre-

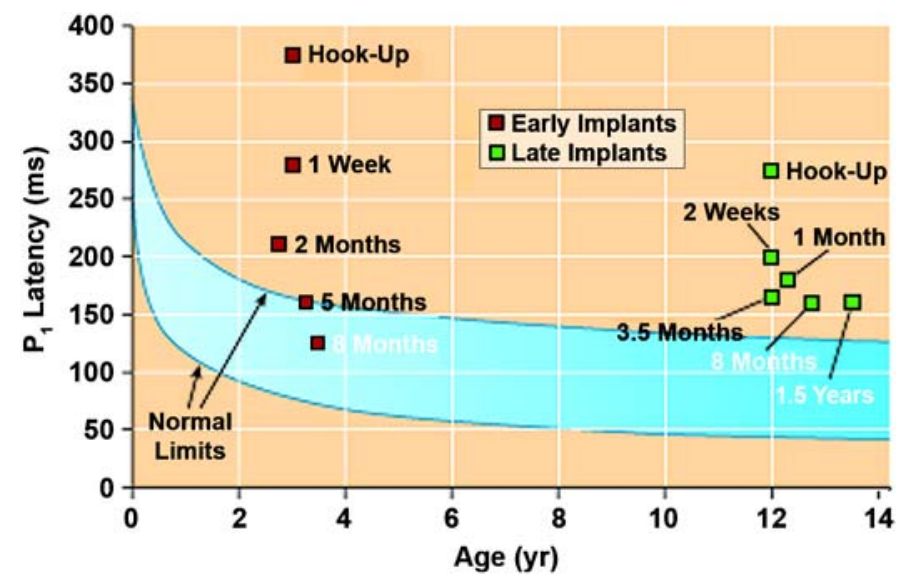

Figure 11.

Latencies of cortical responses ( $\mathrm{P}_{1}$ wave of cortical evoked potential) to brief speech sound for implanted children and children with normal hearing. Latencies for children implanted before age 4 are shown by red squares, and latencies for children implanted at later ages are shown by green squares. Ninety-five percent confidence limits of latencies for 124 children with normal hearing are depicted by solid lines and area filled with blue. Source: Reprinted with permission from Dorman MF, Wilson BS. The design and function of cochlear implants. Am Scientist. 2004;92(5):436-45.

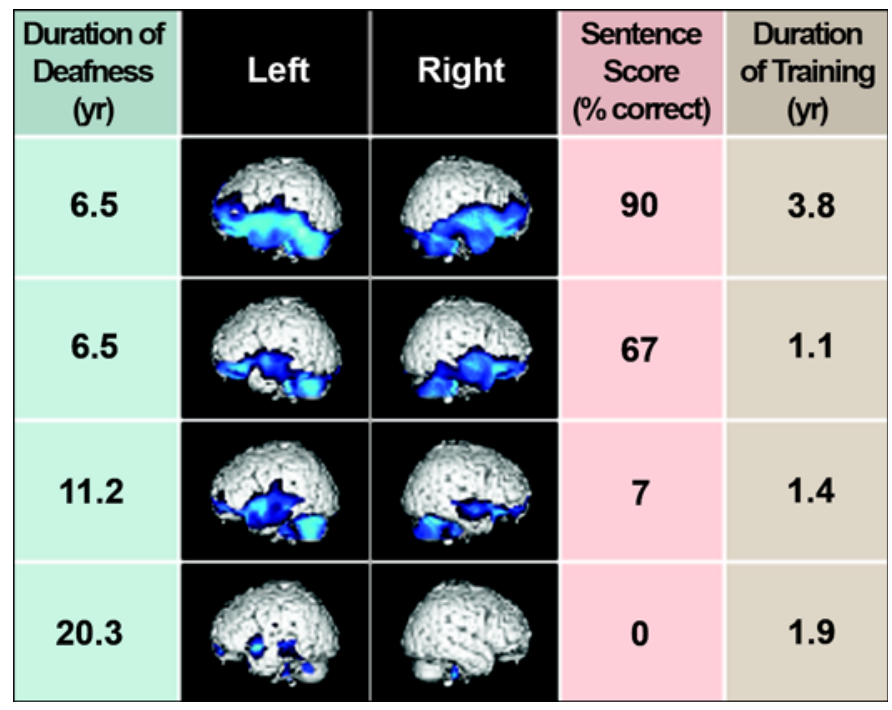

Figure 12.

Differences in metabolic activity in cortical areas prior to implant for successful and relatively unsuccessful users of cochlear implants. Measures were made using positron emission tomography (PET), and subjects were 10 prelingually deaf children ranging in age from 2 to 20 years. Four representative cases are shown. Blue highlighting in brain scans indicates lower-than-normal activity. Progressively lighter shades of blue indicate progressively lower levels of activity. Duration of deafness prior to implant and sentence test score obtained following indicated period of experience and training with implant are also presented for each of four cases in figure. Source: Reprinted with permission from Dorman MF, Wilson BS. The design and function of cochlear implants. Am Scientist. 2004;92(5):436-45. PET images from Lee DS, Lee JS, Ph SH, Kim SK, Kim JW, Chung JK, Lee MC, Kim CS. Cross-modal plasticity and cochlear implants. Nature. 2001;409(6817):149-50 [PMID: 11196628]

sents results from a study conducted by Dong Soo Lee and coworkers at Seoul National University in Seoul, Korea [76].

Figure 11 shows latencies of cortical responses (the $\mathrm{P}_{1}$ wave of the cortical evoked potential) to a brief speech sound for normal and implanted children (data from Sharma et al. [82]). A short latency may indicate fully intact and functional pathways from the cochlea to the cortex. Congenitally deaf children implanted before the age of 4 (red squares) exhibit a substantial reduction in latencies with increasing experience with the implant. On average, at 5 months of experience, the latency enters the normal range (indicated by the blue area in the graph). In contrast, congenitally deaf children implanted later in life (green squares) show some reduction in latencies with experience, but the magnitude of the effect is much smaller than that seen for the early-implanted children and the averages of latencies never enter the normal range, 
even with prolonged experience out to 1.5 years. (Indeed, downward shifts in latency seem to cease at 3.5 months of experience for the late-implanted children.) These results taken together suggest that (1) the brain is more plastic at the earlier ages and (2) once those earlier ages are exceeded, reinstatement of normal latencies is very difficult, at least with current prostheses and standard training procedures.

Figure 12 shows differences in cortical (metabolic) activity prior to implantation for successful and relatively unsuccessful users of cochlear implants (data from Lee et al. [76]). The measures were made using positron emission tomography (PET), and the subjects were 10 prelingually deaf children, ranging in age from 2 to 20 years. The figure presents PET scans for four representative cases, along with the duration of deafness and the score from a test of sentence recognition for each of the cases. All 10 children trained with the implant for at least 8 months prior to the administration of the sentence test. The blue highlighting in the brain scans indicates lowerthan-normal levels of activity, with reference to agematched controls. (The lowest levels are indicated by the lightest shades of blue.) Children with high sentence scores following their implants had shorter durations of deafness and also had large and pronounced regions of hypoactivity in cortical areas normally subserving auditory function (many blue and light blue areas), whereas children with low scores had long durations of deafness and normal or nearly normal levels of activity in these same cortical areas (few blue areas). These findings have been interpreted as evidence of early cross-modal plasticity for the long-deafened children. In particular, quiescent brain regions normally subserving auditory function are recruited or encroached by other sensory modalities (possibly vision or somatosensory inputs) early in life, and this plastic change cannot be reversed or at least not easily reversed later in life, presumably after the expiration of a "sensitive period" for cortical organization (or reorganization). The findings also suggest that the availability and plasticity of the cortex in young recipients of cochlear implants may be the basis for their better scores on the sentence tests.

The brain is likely to be the "tail that wags the dog" in determining outcomes with present-day cochlear implants. The brain "saves us" in achieving high scores with implants by somehow utilizing a crude, sparse, and distorted representation at the periphery. In addition, strong learning or accommodation effects-over long periods ranging from about 3 months to 1 year or moreindicate a principal role of the brain in reaching asymptotic performance with implants (Figure 9). Multiple lines of evidence further indicate or suggest that impairments or changes in brain function-including damage to the auditory pathways in the brain stem, compromised function in the areas of cortex normally devoted to auditory processing, reduced cortical plasticity, or crossmodal plasticity — can produce highly deleterious effects on results obtained with cochlear implants.

\section{Likely Limitations Imposed by Present-Day Electrode Designs and Placements}

Present-day designs and placements of electrodes for cochlear implants do not support more than four to eight effective sites of stimulation, or effective or functional channels, as mentioned previously. Contemporary cochlear implants use between 12 and 22 intracochlear electrodes, so the number of electrodes exceeds the number of effective channels (or sites of stimulation) for practically all patients and for all current devices. The number of effective channels depends on the patient and the speech reception measure used to evaluate performance. For example, increases in scores with increases in the number of active electrodes generally plateau at a lower number for consonant identification than for vowel identification. (This finding makes sense from the perspective that consonants may be identified with combinations of temporal and spectral cues, whereas vowels are identified primarily or exclusively with spectral cues that are conveyed through independent sites of stimulation.) Patients with low speech reception scores generally do not have more than four effective channels for any test, whereas patients with high scores may have as many as eight or slightly more channels depending on the test $[13,84]$.

Results from studies using acoustic simulations of implant processors and subjects with normal hearing indicate that a higher number of effective channels or sites of stimulation for implants could be beneficial. Dorman et al. found, for example, that with the simulations and normal-hearing subjects, as many as 10 channels are needed to reach asymptotic performance (for difficult tests) using a CIS-like processor [85]. Other investigators have found that even more channels are needed for asymptotic performance, especially for difficult tests such as identification of vowels or recognition of speech presented in competition with noise or multitalker babble $[13,86]$. For example, Friesen et al. found that for listeners with 
normal hearing, identification of vowels continued to improve with the addition of channels in the acoustic simulations up to the tested limit of 20 channels, for vowels presented in quiet and at progressively worse $\mathrm{S} / \mathrm{Ns}$ out to and including $+5 \mathrm{~dB}$ [13].

This apparent limitation with present-day cochlear implants is illustrated in Figure 13, which shows speech reception scores as a function of the number of stimulated electrodes (and associated channels) for CIS processors. Figure 13(a) shows results from Prof Wilson's laboratory, and Figure 13(b) shows results from studies conducted by Dr. Carolyn Garnham and coworkers in the United Kingdom [14]. These results typify results from other studies.

Figure 13 shows improvements in speech reception scores - for a variety of tests - with increases in electrode number up to a relatively low value depending on the test. Scores for tests of consonant identification in a quiet condition "saturate" or plateau at three electrodes (Figure 13(a)), and scores for identification of consonants presented in competition with noise at the $\mathrm{S} / \mathrm{N}$ of $+5 \mathrm{~dB}$ saturate or plateau at four (Figure 13(b)) or five (Figure 13(a)) electrodes. Scores for recognition of sentences or vowels also presented in competition with noise at the S/Ns of +10 and $-10 \mathrm{~dB}$, respectively, saturate at six electrodes (Figure 13(b)). Scores for the remaining two tests shown in Figure 13(b) do not increase significantly with increases in electrode number beyond six. These saturation points are well below the maximum number of electrodes for each of the studies, 22 for Figure 13(a) and 10 or 11 (among the available 12 in the implant device used) for Figure 13(b).

Large improvements in the performance of cochlear implants might well be obtained with an increase in the number of effective sites of stimulation, which would help narrow the gap between implant patients and subjects with normal hearing. This gap is especially wide for the many patients who do not have more than four functional channels across wide ranges of speech reception measures. Just a few more channels for the top performers with implants would almost without doubt help them in listening to speech in demanding situations, such as speech presented in competition with noise or other talkers. An increase in the number of functional channels for patients presently at the low end of the performance spectrum could improve their outcomes considerably.

A highly plausible explanation for the limitation in effective channels with implants is that the electric fields from different intracochlear electrodes strongly overlap
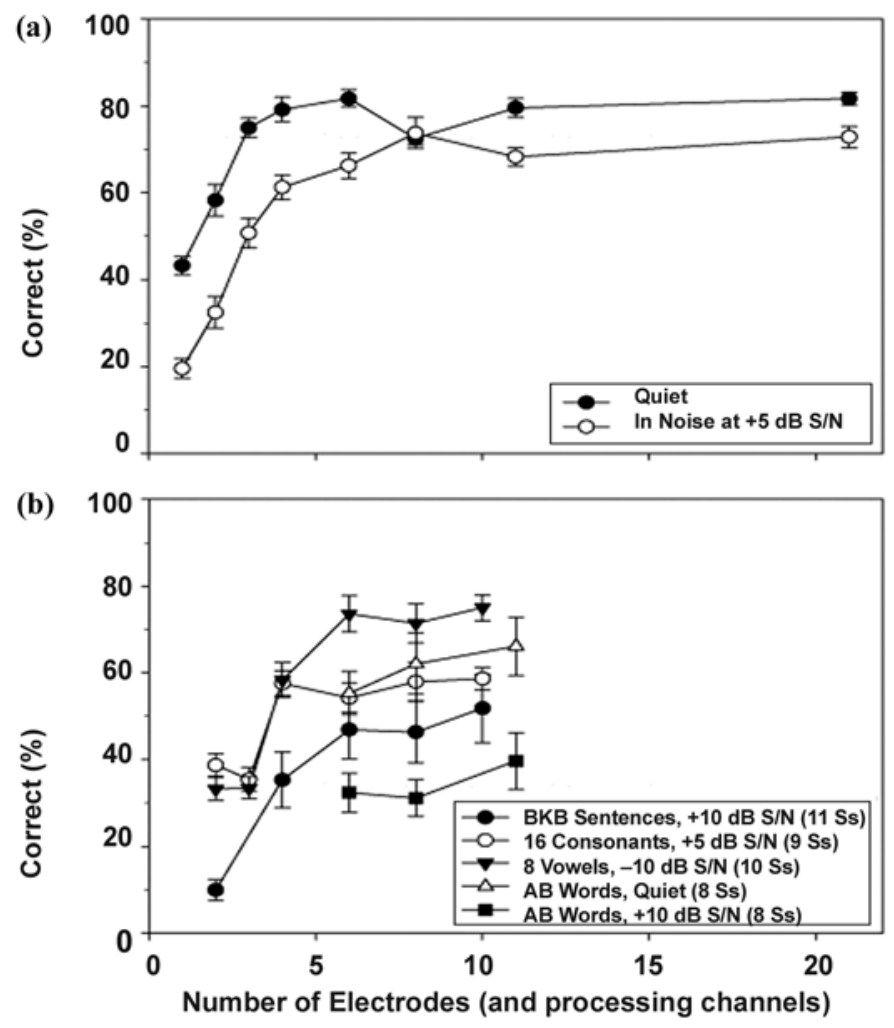

Figure 13.

Speech reception scores as function of number of stimulated electrodes (and associated channels) using continuous interleaved sampling (CIS) processing strategy. Means and standard error of the means are shown. (a) Results from studies conducted in Prof Wilson's laboratory and (b) results from Garnham et al. (Garnham C, O’Driscoll M, Ramsden $\mathrm{R}$, Saeed S. Speech understanding in noise with a Med-El COMBI 40+ cochlear implant using reduced channel sets. Ear Hear. 2002;23(6): 540-52. [PMID: 12476091]). (a) Scores for identification of 24 consonants in /a/-consonant-/a/ context (e.g., "aga," "ata," "ana”) for subject NP-8 using Nucleus cochlear implant system with its 22 intracochlear electrodes. Consonants were presented in quiet or in competition with noise at speech-to-noise ratio $(\mathrm{S} / \mathrm{N})$ of $+5 \mathrm{~dB}$. (b) Scores for maximum of 11 subjects (Ss), with each subject using COMBI 40+ cochlear implant system, which has 12 intracochlear electrodes. Tests for these subjects included recognition of Bench, Kowal, and Bamford (BKB) sentences presented in competition with pink noise at $S / N$ of $+10 \mathrm{~dB}$, identification of 16 consonants in /a/-consonant-/a/ context and presented in competition with noise at $\mathrm{S} / \mathrm{N}$ of $+5 \mathrm{~dB}$, identification of 8 vowels in /b/-vowel-/d/ context (e.g., "bad," "bed," "baud”) presented in competition with noise at $\mathrm{S} / \mathrm{N}$ of $-10 \mathrm{~dB}$, and recognition of Arthur Boothroyd (AB) monosyllabic words presented either in quiet or in competition with noise at $\mathrm{S} / \mathrm{N}$ of $+10 \mathrm{~dB}$. Number of subjects taking each test is indicated within parentheses for each line in legend. Additional experimental conditions for study depicted in (a) are same as those described in Wilson BS. The future of cochlear implants. Br J Audiol. 1997;31(4):205-25. [PMID: 9307818]. Additional experimental conditions for study depicted in (b) are presented in Garnham et al. 
at the sites of neural excitation $[84,87]$. Such overlaps (or electrode interactions) may well impose an upper bound on the number of electrodes that are sufficiently independent to convey perceptually separate channels of information. In addition, a central processing deficit may contribute to the limitation, perhaps especially for patients with low speech reception scores and (usually) a relatively low number of effective channels.

A problem with ST implants is that the electrodes are relatively far from the target tissue (the spiral ganglion), even for placements of electrodes next to the inner wall of the ST. Close apposition of the target and the electrode is necessary for a high spatial specificity of stimulation [88]. One possibility for providing a close apposition is to promote the growth of neurites from the ganglion cells toward the electrodes in the ST with controlled delivery of neurotrophic drugs into the perilymph [89-92]. Such growth of neurites would bring the target to the electrodes. Another possibility is to implant an array of electrodes directly within the auditory nerve (an intramodiolar implant) through an opening made in the basal part of the cochlea [19-21,23-25]. In this case, the electrodes would be placed immediately adjacent to axons of the auditory nerve. Studies are underway to evaluate each of these possibilities, including safety and efficacy studies. Results from studies evaluating the intramodiolar implant have demonstrated that it is feasible from fabrication and surgical perspectives and that the number of independent sites of stimulation with that implant may be substantially higher than the number for ST implants [24-25]. However, these are preliminary findings and a complete course of safety studies needs to be completed before intramodiolar implants might be approved by the U.S. Food and Drug Administration (and other regulatory agencies worldwide) for applications in humans. The same is true for the use of neurotrophic drugs to promote the growth of neurites toward ST electrodes. Each of these possibilities is promising, but each needs further study and validation.

\section{RECENT ADVANCES}

Two recent advances in the design and performance of cochlear implants are (1) electrical stimulation of both ears with bilateral cochlear implants and (2) combined electric and acoustic stimulation (EAS) of the auditory system for persons with residual hearing at low frequencies. Bilateral electrical stimulation may reinstate, at least to some extent, the interaural amplitude and timing difference cues that allow people with normal hearing to lateralize sounds in the horizontal plane and to selectively "hear out" a voice or other source of sound from among multiple sources at different locations. Additionally, stimulation on both sides may allow users to make use of the acoustic shadow cast by the head for sound sources off the midline. In such cases, the $\mathrm{S} / \mathrm{N}$ may well be more favorable at one ear than the other for multiple sources of sound and users may be able to attend to the ear with the better S/N. EAS patients with only mild to moderate elevations in low-frequency hearing thresholds may benefit from a frequency-appropriate representation of pitch (F0) and an adequate representation of low-frequency format peaks. This information, if present, would complement the higher frequency information provided by the cochlear implant and electrical stimulation. Various surgical techniques and drug therapies have been developed to preserve low-frequency hearing in an implanted cochlea, including (1) deliberately shallow insertions of the electrode array $(6,10,16$, or $20 \mathrm{~mm})$ so as not to damage the apical part of the cochlea and remaining hair cells there, (2) insertion of the electrode array through the round window membrane rather than through a cochleostomy to eliminate deleterious effects of drilling (loud and possibly damaging levels of noise, introduction of blood and bone dust into the perilymph, possible damage to delicate cochlear structures such as the BM), (3) use of "soft surgery" techniques to minimize trauma, (4) use of thin and highly flexible electrodes, (5) use of a lubricant such as hyaluronic acid to facilitate insertion of the array, and (6) use of corticosteroids and other drugs to help preserve cochlear structures in the face of surgical manipulations and the introduction of a foreign body into the inner ear. Moderate-to-excellent preservation of residual hearing has been reported for a majority of patients using the shallow insertions and some or all of the additional procedures and techniques just mentioned [93-107], although residual hearing is still completely lost for some patients with the same insertions and approaches. Among the tested methods, insertion through the round window for placement of $20 \mathrm{~mm}$ arrays or use of shorter arrays appear to be especially effective [103,106,108-109]. The "soft surgery" methods also have been identified as important [102,110]. Studies aimed at the further development of surgical techniques, adjunctive drug therapies, and special electrode arrays are in progress. 
Each of these approaches - bilateral electrical stimulation and combined EAS-has produced large improvements in speech reception performance compared with control conditions. In particular, bilateral stimulation can provide a substantial benefit for recognizing speech presented in competition with spatially distinct noise compared with scores obtained with either unilateral implant alone [63,111-127]. In addition, use of both implants supports an improved ability to lateralize or localize sounds (depending on which was measured in a particular study), again compared with either unilateral implant [113,116117,119-120,124-125,127-133]. (This ability is nonexistent or almost nil with a unilateral implant.) Combined EAS also provides a substantial benefit for listening to speech in quiet, in noise, or in competition with multitalker babble compared with either electric or acoustic stimulation alone [63,93-94,96-97,99,101-105,134-140]. Indeed, in some cases, the score for combined EAS is greater than the sum of the scores for the electric- and acoustic-only conditions. This finding has been described as a synergistic effect $[63,97,101,134,141]$. In addition, identification of melodies and reception of musical sounds is greatly improved with combined EAS compared with electric stimulation alone [99,104,137,140,142-143]. (Scores with acoustic stimulation alone closely approximate the scores with combined EAS for melody and music reception.) In cases of symmetric or nearly symmetric hearing loss, the benefits of combined EAS can be obtained with the acoustic stimulus delivered either to the ear with the cochlear implant or to the opposite ear or to both ears [134]. Large benefits also can be obtained in cases of complete or nearly complete loss of residual hearing on the implanted side and delivery of the acoustic stimulus to a still-sensitive ear on the contralateral side [137,139,140,144-145]. (This observation is good news for recipients of a fully inserted cochlear implant on one side and residual hearing on the contralateral side, in that any residual hearing on the implanted side is generally lost with a full insertion of the electrode array.)

The described gains from bilateral electrical stimulation most likely arise from a partial or full restoration of the binaural difference cues and the head shadow effect, as suggested previously. In addition, gains may result from a "binaural summation" effect that is produced in normal hearing by redundant stimulation on the two sides. Detailed descriptions of these various contributors to an overall binaural benefit for normal hearing and possible contributors for prosthetic hearing are presented in Wilson et al. [63]. The evidence to date indicates that almost all recipients of bilateral cochlear implants benefit from the head shadow effect and that some benefit from (1) the binaural squelch effect that is made possible with presentation and perception of the binaural timing-difference cue, (2) the binaural summation effect, or (3) both. The largest contributor to improvements in listening to speech presented in competition with spatially distinct noise is the head shadow effect, which is a physical effect that is present and can be used whether or not the binaural processing mechanism in the brain stem is intact. (However, some central function must be involved in attending to the ear with the better $\mathrm{S} / \mathrm{N}$, and this appears to be intact for most of the tested recipients of bilateral cochlear implants. This function and its applicability to bilateral cochlear implants is discussed in Tyler et al. [146].)

In addition to these binaural effects that occur in normal hearing and to a variable extent in prosthetic hearing, electric stimulation on both sides may help fill "gaps" in the representation of frequencies on one side-because of uneven survival of spiral ganglion cells along the length of the cochlea-with complementary excitation of surviving neurons at the same frequency place(s) on the contralateral side. For example, a lack of input to the central nervous system (CNS) at the $5 \mathrm{kHz}$ position on one side may be at least partly bridged or compensated for by stimulation of remaining neurons at the $5 \mathrm{kHz}$ position in the other ear. This mechanism and the binaural summation effect may contribute to the large improvements observed with bilateral implants for the recognition of difficult speech material presented from in front of the subjects and without any interfering noise, where the interaural difference cues and the head shadow effect do not come into play. The mechanism also may contribute to the good results observed for other conditions, in which the difference cues and the head shadow effect are also present.

A further possible mechanism contributing to the observed benefits of bilateral electric stimulation is a higher number of effective channels. Bilateral implants in general provide a doubling or near doubling of physical stimulus sites compared with either unilateral implant alone. This increase may provide some gain in the number of effective channels, especially in cases of uneven nerve survival across the two sides, where stimulation of an area on one side that is "dead" on the other side may add an effective channel. As noted before, even a small gain in the number of effective channels could produce a large benefit, particularly for patients who otherwise 
would have low levels of performance and particularly for reception of difficult speech materials or for listening to speech in adverse $\mathrm{S} / \mathrm{N}$ conditions.

An example of findings from studies with recipients of bilateral implants is presented in Figure 14. These results are from studies conducted by Prof Dr. Joachim Müller and coworkers at the Julius-Maximilians Universität in Würzburg, Germany [114]. Nine subjects participated. Figure 14(a)-(b) shows individual and average scores for the recognition of sentences presented in competition with speech-spectrum noise at the $\mathrm{S} / \mathrm{N}$ of $+10 \mathrm{~dB}$, with the sentences presented through a loudspeaker in front of the subject and the noise presented through a loudspeaker to the right of the subject (Figure 14(a)) or to the left of the subject (Figure 14(b)). Figure 14(c) shows results for the recognition of monosyllabic words in quiet presented through a loudspeaker in front of the subject. For the sentence tests, the difference in scores for the left implant only versus the right implant only shows the magnitude of the head shadow benefit, which is large (Figure 14(a)-(b), lower panels). For these same tests, the difference in scores for the bilateral condition versus the score for the single implant at the side opposite to the noise source shows the magnitude of a "binaural processing benefit," which is a combination of binaural squelch, binaural summation, and possibly other effects. This binaural processing benefit is smaller than the head shadow benefit but is still significant. For the word test (Figure 14(c)), the difference in scores between the bilateral condition and either of the unilateral conditions may be attributable to a binaural summation effect, a filling of gaps in nerve survival across the two sides, a principal contribution from the better of the two ears, a higher number of effective channels, or some combination of these. The improvement obtained with stimulation on both sides is large (see dark gray bars in Figure 14), comparable in magnitude to the head shadow benefits demonstrated by the results from the sentence tests. This improvement is larger than what would be expected from binaural summation effects alone.

Among the possible mechanisms just mentioned, the "better-ear effect" may provide almost half of the improvement observed with bilateral stimulation and with a single source from in front of the subject. Figure 15 compares the score that would have been obtained if the subject could have attended to the better of the two ears only (gray bar) versus the scores presented previously in the lower panel of Figure 14(c), showing the average scores across subjects for bilateral stimulation and for stimulation of either unilateral implant alone (dark gray bars). As can be seen, the increase in scores over either unilateral condition in attending to the better ear only for each subject is about 40 percent of the total improvement produced with bilateral stimulation. Other factors, such as binaural summation or a filling of gaps in nerve survival across the two sides, must account for the remaining difference.

The apparent magnitude of the "better-ear effect" is large. Thus, the guarantee that the better ear is implanted is an important advantage of bilateral cochlear implants, especially in view of the fact that the better ear cannot be predicted or identified prior to surgery (and subsequent fitting and use of the implant system), at least through use of present preoperative measures [113,126]. (The better-ear effect may also produce improvements in the overall performance of implant systems for populations of patients, including performance for listening to single sources off the midline and for listening to speech in competition with noise or other talkers, such as the conditions presented in Figure 14(a)-(b). These contributions to performance would be expected to be smaller than those illustrated in Figure 15 for the ideal condition, but nonetheless may still be significant.)

The described gains from combined EAS may arise from a normal or nearly normal input to the CNS for lowfrequency sounds from the acoustic stimulation in conjunction with a crude representation of higher frequency sounds from the electric stimulation with a partially or fully inserted cochlear implant. (In the case of a partially inserted implant, the acoustic stimulus may be delivered to the ipsilateral side, the contralateral side, or both sides; in the case of a fully inserted implant, the acoustic stimulus usually must be delivered to the contralateral side only.) The CNS apparently is able to integrate these seemingly disparate inputs from the two modes of stimulation and from generally different regions of the cochlea into a single auditory percept that is judged by patients as sounding natural and intelligible.

A principal advantage of combined EAS may be that FS information is presented without modification in the low-frequency range and a substantial portion or all of this information may be perceived, at least by the better users. The FS information is likely to include F0s and the first one or two harmonics of the F0s, along with at least some indication of first formant frequencies for speech. The information is also likely to include most F0s and perhaps the first one or two harmonics (depending on the 
(a)
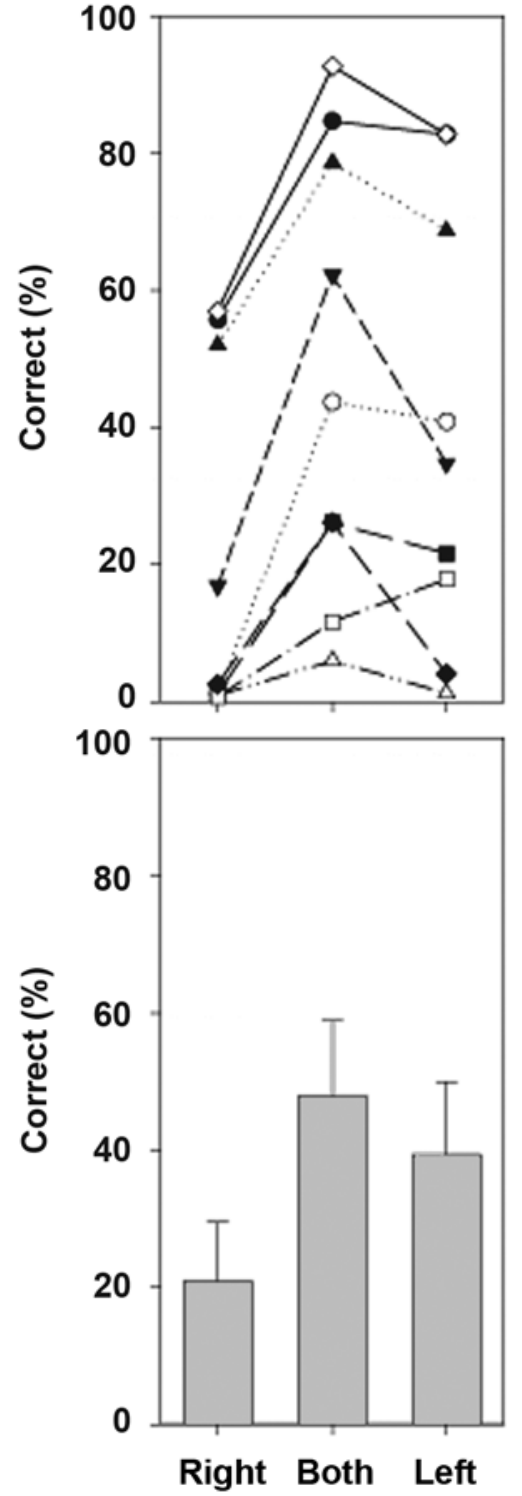

(b)
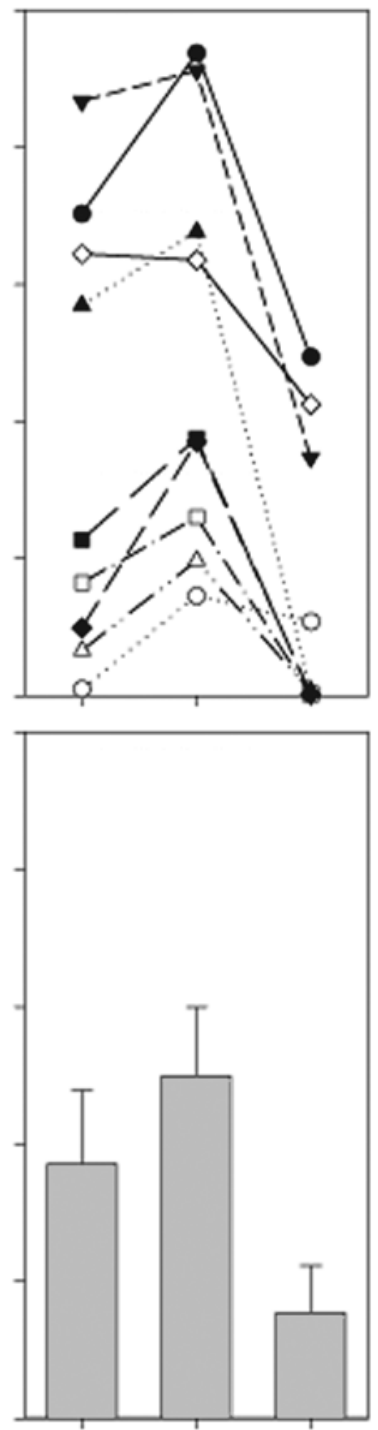

Right Both Left (c)

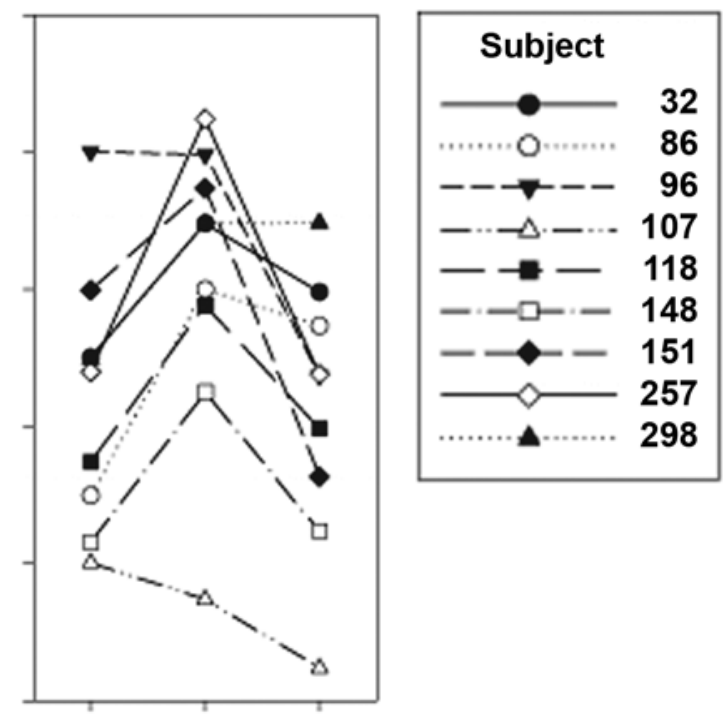

Implant (Side)

Figure 14.

Results from studies conducted by Müller et al., with nine recipients of bilateral cochlear implants (Müller J, Schön F, Helms J. Speech understanding in quiet and noise in bilateral users of the MED-EL COMBI 40/40+ cochlear implant system. Ear Hear. 2002;23(3):198-206. [PMID: 12072612]). (a) Speech reception scores for individual subjects (top) and means and standard error of the means (bottom) for identification of words in HochmairSchultz-Moser (HSM) sentences presented in competition with Comité Consultatif International Téléphonique et Télégraphique (CCITT) speechspectrum noise at speech-to-noise ratio $(\mathrm{S} / \mathrm{N})$ of $+10 \mathrm{~dB}$ with noise presented from loudspeaker $1 \mathrm{~m}$ to right of subject. (b) Speech reception scores for individual subjects (top) and means and standard error of the means (bottom) for identification of words in HSM sentences presented in competition with CCITT speech-spectrum noise at $\mathrm{S} / \mathrm{N}$ of $+10 \mathrm{~dB}$ with noise presented from loudspeaker $1 \mathrm{~m}$ to left of subject. (c) Speech reception scores for individual subjects (top) and means and standard error of the means (bottom) for recognition of Freiburger monosyllabic words presented in quiet. Each panel shows scores obtained with right implant only, both implants, and left implant only. Speech was presented from loudspeaker $1 \mathrm{~m}$ in front of subject for all tests. Panel with dark gray bars indicates efficacy of bilateral stimulation even for conditions without interfering noise and in absence of binaural difference cues. Source: Wilson BS, Lawson DT, Müller JM, Tyler RS, Kiefer J. Cochlear implants: Some likely next steps. Annu Rev Biomed Eng. 2003;5:207-49. [PMID: 12704085]. Reprinted, with permission, from the Annual Review of Biomedical Engineering, Vol. 5. (C) 2003 by Annual Reviews (www.annualreviews.org). 


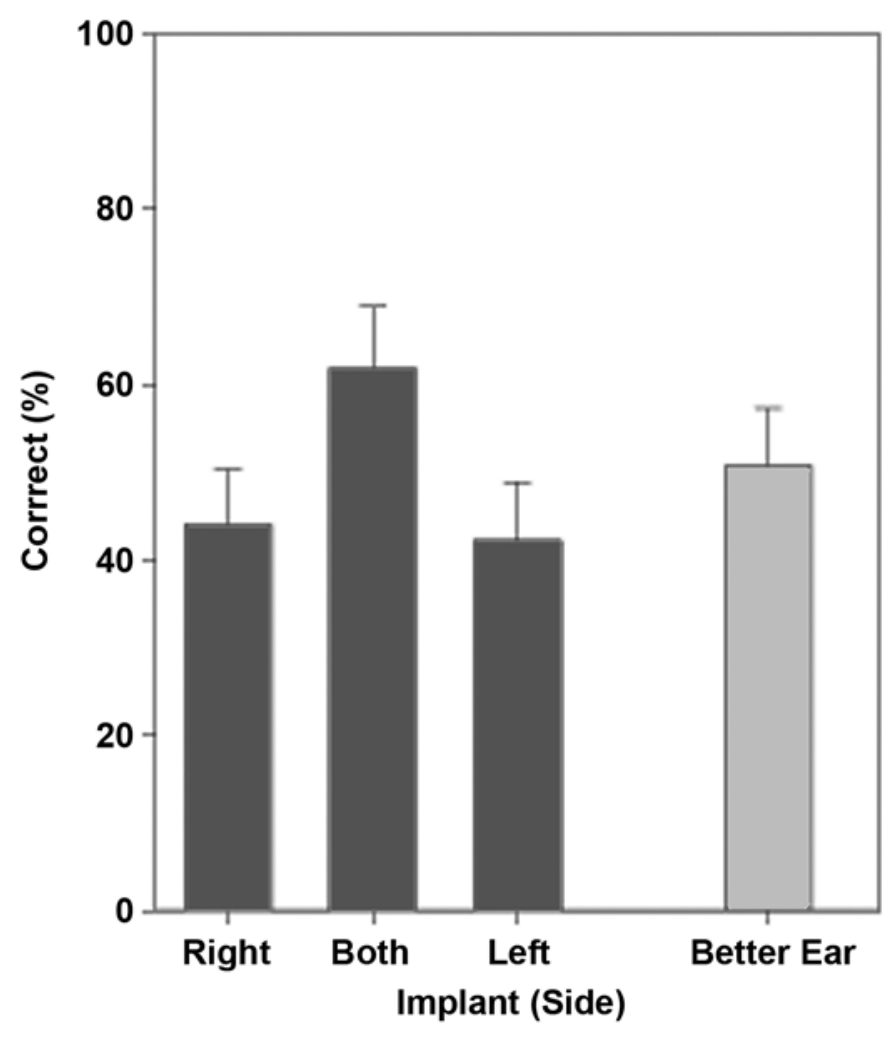

Figure 15.

Repetition of data presented in bottom panel of Figure 14(c) (dark gray bars), along with mean and standard error of the mean for better of two unilateral scores shown in top panel of Figure 14(c). Better-ear results shown by light gray bar and associated error bar. Data from studies conducted by Müller et al., with nine recipients of bilateral cochlear implants (Müller J, Schön F, Helms J. Speech understanding in quiet and noise in bilateral users of the MED-EL COMBI 40/40+ cochlear implant system. Ear Hear. 2002;23(3):198-206. [PMID: 12072612]).

F0) for music. This representation of FS information with combined EAS may be more natural and more effective than (the necessarily limited) representations of the information using electric stimuli, as outlined previously in the section "Transformation of Microphone Inputs into Stimuli for Cochlear Implants.” The representation provided by combined EAS can only be effective for the lowfrequency range, of course, but FS information in this range is more important than FS information at higher frequencies for both speech and music reception [38].

Some investigators have suggested that an ability to separate different "auditory streams" on the basis of different F0s (and trajectories of F0s) for different sounds may be the basis for the good results obtained with combined EAS for speech reception tasks [103,136-137,147-148]. In particular, the F0s are presented in the acoustic-stimulation part of combined EAS, and the user may be able to perceive those frequencies with far greater accuracy when using residual, low-frequency hearing than with electrically evoked hearing, even with a fully inserted cochlear implant. Perception of small differences in frequencies for frequencies in the typical range of the residual hearing, i.e., below 500 to $1,000 \mathrm{~Hz}$, may allow for an effective separation of a signal from interfering sounds. This ability would help in conditions where speech is presented in competition with noise or other talkers, especially one other talker. Such perception could also explain the large benefits of combined EAS for listening to music [143], in that much of the melodic information in music is conveyed by F0s below 500 to $1,000 \mathrm{~Hz}$.

A problem with this idea-at least for speech reception in the presence of competing sounds-is that large gains also are observed with combined EAS for speech presented in quiet conditions, including monosyllabic words presented in quiet and in isolation. It is difficult to imagine how a better perception of F0s could help in this situation. Possibly, multiple mechanisms are at play, or possibly, some other (common) mechanism may underlie all of the observed effects, such as reception of most or all of the FS information that is presented in the lowfrequency range (and not just the F0s). In any case, the mechanism or mechanisms producing the benefits remain(s) to be unequivocally identified [140].

Examples of findings from studies to evaluate combined EAS are presented in Figures 16 and 17. Both figures show data from Prof Dorman's laboratory [140] and are consistent with data obtained elsewhere and as cited previously. Figure 16 shows mean scores and standard deviations for a variety of speech reception measures and for 15 subjects with a fully inserted cochlear implant on one side and residual low-frequency hearing on the contralateral side. In that contralateral ear, the mean thresholds at $500 \mathrm{~Hz}$ and lower for these subjects were $53 \mathrm{~dB}$ hearing level (HL) and better, and the mean thresholds at $1 \mathrm{kHz}$ and above were $81 \mathrm{~dB}$ HL and worse. This is a typical pattern of hearing loss for many people, i.e., a "ski slope" or "corner audiogram" loss, and fulfills the criteria for combined EAS for ipsilateral (with a partially inserted implant) acoustic stimulation as well as the applied contralateral stimulation. The open bars in Figure 16 show scores for acoustic stimulation alone, delivered to the ear contralateral to the cochlear implant; the light gray bars show scores for electric stimulation alone; and the dark gray bars 

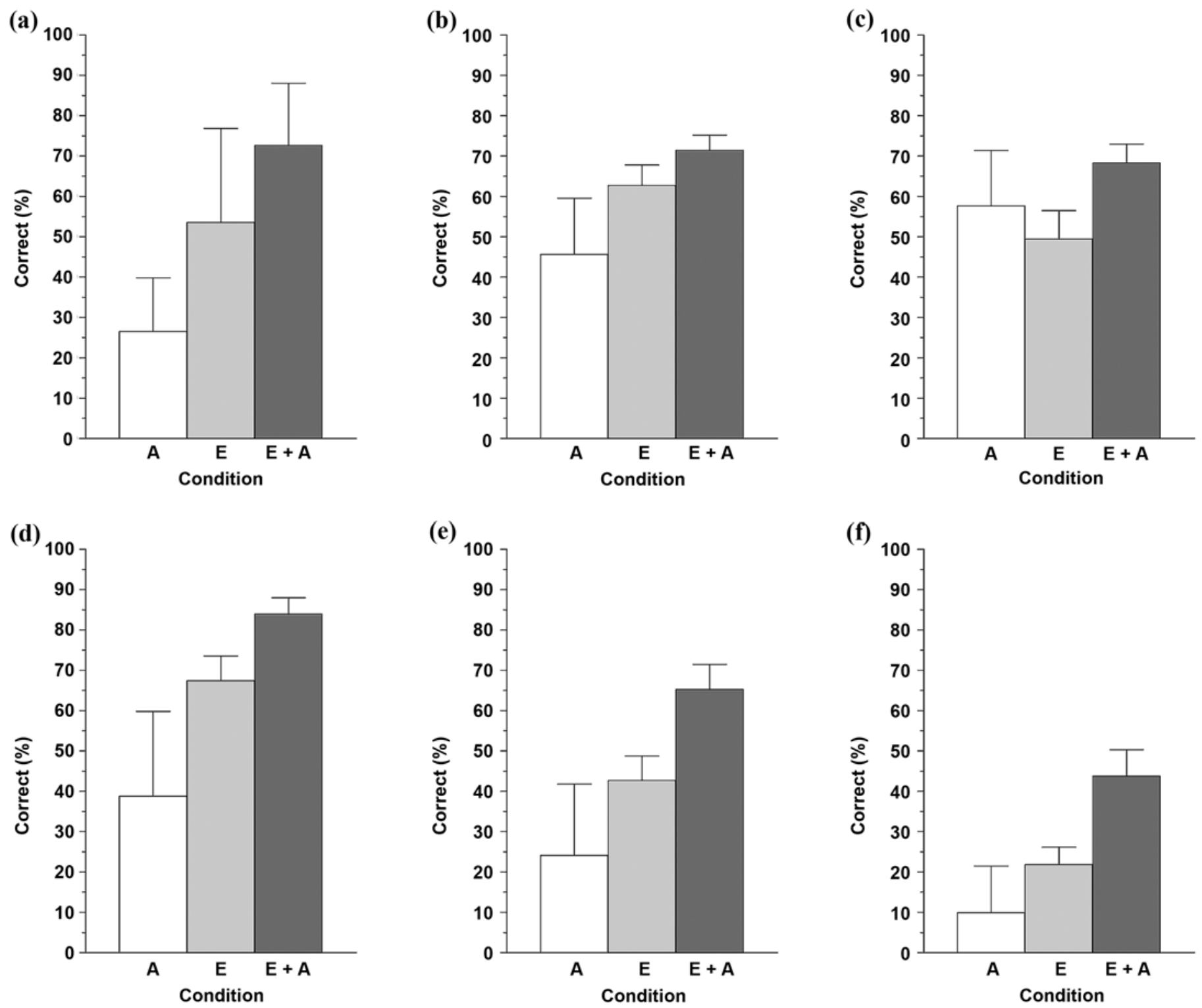

Figure 17.

Mean and standard deviation for electric stimulation only (E), acoustic stimulation only (A), and combined electric and acoustic stimulation (E + A) conditions from Dorman et al. (see source). Tests included (a) recognition of consonant-nucleus-consonant words presented in quiet, (b) identification of consonants in /e/-consonant-/e/ context (e.g., "a bay,” “a day,” "a gay”), (c) identification of 13 synthesized vowels in /b/-vowel-/t/ context (e.g., "bait," "Bart," "bat”) with equal durations to eliminate any temporal cues, and recognition of Arizona Biomedical sentences presented in (d) quiet or in competition with four-talker babble at speech-to-babble ratios of (e) $+10 \mathrm{~dB}$ and (f) $+5 \mathrm{~dB}$. Source: Reprinted with permission of S. Karger AG, Basel from Dorman MF, Gifford RH, Spahr AJ, McKarns SA. The benefits of combining acoustic and electric stimulation for the recognition of speech, voice and melodies. Audiol Neurootol. 2008;13(2):105-12. [PMID: 18057874]

show scores for the combined EAS condition. The measures included recognition of CNC monosyllabic words, identification of consonants in an /e/-consonant-/e/ context (e.g., "a bay," "a day,” "a gay”), identification of 13 synthesized vowels in a /b/-vowel-/t/ context (e.g., "bait," "Bart," "bat") and with equal durations to eliminate any temporal cues, and recognition of the AzBio sentences [149] presented in quiet or in competition with a fourtalker babble at the $\mathrm{S} / \mathrm{Bs}$ of +10 and $+5 \mathrm{~dB}$.

The results demonstrate large benefits of combined EAS. Analyses of the variance indicate significant differences among the three conditions for each of the tests 
JRRD, Volume 45, Number 5, 2008

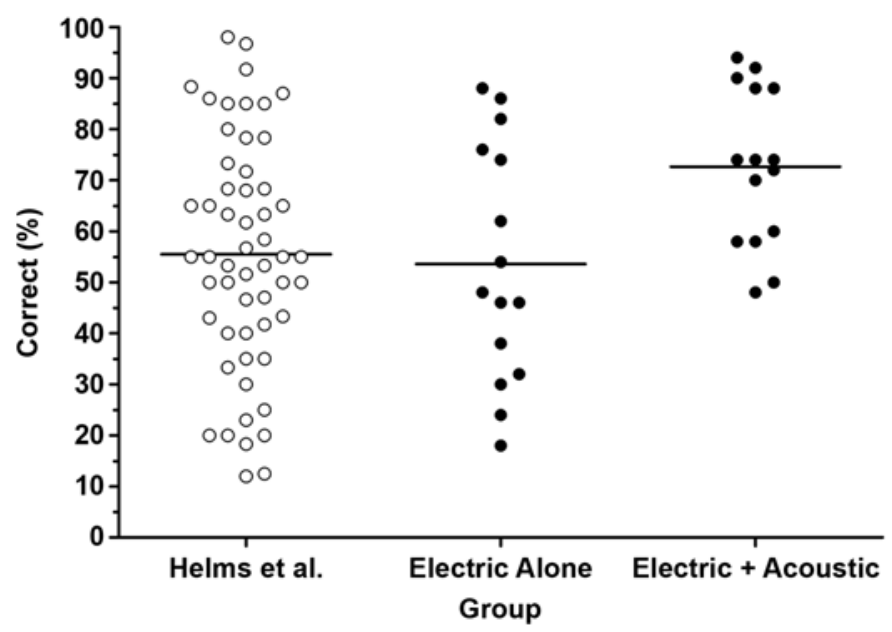

Figure 16.

Individual scores for monosyllabic word test in Figure 16 from study by Dorman et al. (see source). Scores for electric stimulation only condition are presented in middle column, and scores for combined electric and acoustic stimulation condition are presented in right column. Scores from Helms et al. study also are presented in left column for reference (Helms J, Müller J, Schön F, Moser L, Arnold W, Janssen T, Ramsden R, Von Ilberg C, Kiefer J, Pfennigdorf T, Gstöttner W, Baumgartner W, Ehrenberger K, Skarzynski H, Ribari O, Thumfart W, Stephan K, Mann W, Heinemann M, Zorowka P, Lippert KL, Zenner HP, Bohndord M, Hüttenbrink K, Hochmair-Desoyer I, et al. Evaluation of performance with the COMBI40 cochlear implant in adults: A multicentric clinical study ORL J Otorhinolaryngol Relat Spec. 1997;59(1):23-35. [PMID: 9104746]). These scores are same as those shown in rightmost column of Figure 8(b). Subjects in Helms et al. study used unilateral cochlear implant and received electric stimulation only. Horizontal lines in present figure show means of scores for each of three conditions. Source: Reprinted with permission of S. Karger AG, Basel from Dorman MF, Gifford RH, Spahr AJ, McKarns SA. The benefits of combining acoustic and electric stimulation for the recognition of speech, voice and melodies. Audiol Neurootol. 2008;13(2):105-12. [PMID: 18057874]

except for vowel recognition. Results from post hoc tests indicate significant differences between any pair of conditions for the CNC word test and for all three sentence tests. Results from the post hoc tests also indicate a significant difference between the acoustic alone and EAS conditions for the consonant test. Note that significant increases in scores are observed between the electric alone and EAS conditions for speech material presented in quiet (CNC words, sentences in quiet), as well as speech material presented in competition with the four-talker babble (sentences at $+10 \mathrm{~dB}$ and $+5 \mathrm{~dB} \mathrm{~S} / \mathrm{B}$ ). A synergistic effect-in which the EAS score is greater than the sum of the scores for the electric alone and acoustic alone conditions-is seen for the most adverse condition, sentences presented in competition with speech babble at the $\mathrm{S} / \mathrm{B}$ of $+5 \mathrm{~dB}$. In all, these improvements are impressive.

Figure 17 shows the individual scores of the 15 subjects for the CNC word test. Scores for the electric-only condition are shown in the middle column, and scores for the combined EAS condition are shown in the right column. In addition, scores for the 55 subjects in the Helms et al. study [57] are shown in the left column to provide a reference for findings with a fully inserted cochlear implant from a large population of tested subjects. (These data are the same as those presented in the rightmost column of Figure 8(b). The appearance of the data between the figures is somewhat different, since the individual scores are plotted with a greater horizontal displacement in Figure 17 than in Figure 8.) The mean of the scores in each of the columns in Figure 17 is indicated with a horizontal line.

Comparison of the left and middle columns in Figure 17 indicates that the average performance and the distribution of scores for the 15 subjects in the Dorman et al. study (middle column) closely approximate those measures for the larger Helms et al. study (left column). Thus, the 15 subjects have performances with electric stimulation only that are fully representative of performances with contemporary cochlear prostheses and with a much larger population of subjects.

Comparison of the middle and right columns shows that, while the mean of the scores increases with the combined EAS condition, the top scores remain about the same between the two conditions. That is, the top performers with electric stimulation only may be receiving the same or equally effective information as the top performers with combined EAS. This effect was also seen for comparisons between the 15 subjects of the Dorman et al. study and a large population of implant patients in the United States who had CNC word scores of 50 percent correct or better, i.e., top performers with conventional unilateral implants (65 subjects). The comparisons included all of the tests shown in Figure 16. The mean scores, top scores, and distributions of scores between the populations were all quite similar (and not statistically different) for each of the tests, including the sentence test at the $+5 \mathrm{~dB} S / \mathrm{B}$, which was not subject to possible ceiling effects. Thus, unique —or uniquely useful—information is either not presented or received by the patients for the combined EAS condition. Instead, the condition may provide information that is missing or incomplete for patients who have relatively low scores for the electriconly condition. In such cases, combined EAS provides a 
substantial benefit, as shown in the right column of Figure 17; i.e., the lowest scores in the middle column of the figure (electric stimulation only) are brought up to much higher levels in the right column (combined EAS). Indeed, the lowest scores for the EAS condition approximate the mean for the electric-only condition. This is a tremendous improvement and shows that the proportion of patients with high scores is much greater with combined EAS than with electric stimulation only.

Each of these relatively new approaches, bilateral electrical stimulation and combined EAS, utilizes or reinstates a part of the natural system. Two ears are better than one, and use of even a part of normal or nearly normal hearing at low frequencies can provide a highly significant advantage.

\section{POSSIBILITIES FOR FURTHER IMPROVEMENTS}

Great progress has been made in the design and performance of cochlear prostheses. However, much room remains for improvements. Patients with the best results still do not hear as well as listeners with normal hearing, particularly in demanding situations such as speech presented in competition with noise or other talkers. Users of standard unilateral implants do not have good access to music and other sounds that are more complex than speech. Most importantly, speech reception scores still vary widely across patients for relatively difficult tests, such as recognition of monosyllabic words, with any of the implant systems now in widespread use.

Fortunately, major steps forward have been made recently and many other possibilities for further improvements in implant design and function are on the horizon. Electrical stimulation on both sides with bilateral cochlear implants and combined EAS for persons with some residual hearing have been described. These are new approaches, which may well be refined or optimized for still higher levels of performance. Some of the possibilities for such improvements are just now being explored, as are ways to preserve residual hearing in an implanted cochlea. In addition, other approaches-such as (1) reinstatement of spontaneous-like activity in the auditory nerve [150], (2) one or more of the previously described approaches for representing FS information with implants, or (3) a closer mimicking with implants of the processing that occurs in the normal cochlea [33,52] — may also produce improvements in perform- ance, especially for patients with good or relatively good function in the central auditory pathways and in the cortical areas that process auditory information.

Further improvements for all patients might be produced by somehow increasing the number of effective channels supported by cochlear implants. Several possibilities for this have been mentioned, including intramodiolar implants and drug-induced growth of neurites toward the electrodes of ST implants. An additional possibility is to regard bilateral implants as a collection of many stimulus sites and to choose for activation the perceptually separable sites among them. Alternatively, one might "interlace" stimulus sites across the two sides, where the most basal region of one cochlea is stimulated on one side, the next most basal region on the other side, the next most basal region on the first side, and so forth until the full tonotopic map is spanned. In this way, all the frequencies would be represented but the distance between active electrodes in each implant would be doubled, which would in turn reduce the interactions among them compared with stimulation of adjacent electrodes. These different ways of using bilateral implants have the potential to increase the number of effective channels [63,151] but almost certainly at the cost of diminishing or eliminating a useful representation of the binaural difference cues. This may be a good trade-off for some patients.

Each of the approaches described in the preceding two paragraphs is aimed at improving the representation at the periphery. A fundamentally new approach may be needed to help those patients presently at the low end of the performance spectrum, however. They may have compromised "auditory brains" as suggested previously and by many recent findings. For them, a "top down" or "cognitive neuroscience" approach to implant design may be more effective than the traditional "bottom up" approach. In particular, the new (top down) approach would ask what the compromised brain needs as an input in order to perform optimally, in contrast to the traditional approach of replicating insofar as possible the normal patterns of activity at the auditory nerve. The patterns of stimulation specified by the new approach are quite likely to be different from the patterns specified by the traditional approach.

A related possibility that may help all patients at least to some extent is directed training to encourage and facilitate desired plastic changes in brain function (or, to put it another way, to help the brain in its task of learning how to use the inputs from the periphery provided by a cochlear implant). 
Such training, if well designed, may reduce the time needed to reach asymptotic performance and may produce higher levels of auditory function at that point and beyond. The ideal training procedure for an infant or young child may be quite different from the ideal procedure for older children or adults because of differences in brain plasticity. For example, the "step size" for increments in the difficulty of a training task may need to be much smaller for adults than for infants and young children [152]. However, all patients may benefit from appropriately designed procedures that respect the differences in brain plasticity according to age.

The brain is a critical part of a prosthesis system. For patients with a fully intact brain, the bottom up approach to implant design is probably appropriate; i.e., an evercloser approximation to the normal patterns of neural discharge at the periphery is likely to provide the inputs that the brain "expects" and is configured to receive and process. For patients with a compromised brain, such inputs may not be optimal. In those cases, a top down approach to implant design, or a combination of top down and bottom up approaches, may produce the best results. For example, a top down approach combined with techniques to minimize electrode interactions at the periphery may be especially effective for patients presently shackled with poor outcomes.

\section{CONCLUSIONS}

In summary, the experience thus far with cochlear implants indicates or suggests the following:

- A decidedly sparse, crude, and distorted representation at the periphery supports a remarkable restoration of function for some users of present-day cochlear implants. This fortuitous result bodes well for the development of vestibular, visual, or other types of sensory neural prostheses.

- However, this representation must exceed some putative threshold of quality and quantity of information. Most likely, this means that aspects of the normal physiology need to be mimicked or reinstated to some minimal extent. The experience with cochlear implants indicates that (1) not all aspects of the normal physiology need to be reproduced and (2) those aspects that are reinstated do not have to be perfectly reproduced. Present-day implants-with multiple channels of processing; multiple sites of stimulation in the cochlea; and the CIS, $n$-of- $m$, ACE, HiRes, or other modern processing strategies-have exceeded the putative threshold for the great majority of patients, in that most patients score at 80 percent correct or higher in sentence tests using hearing alone and many patients can use the telephone without difficulty. Prior implant systems did not exceed this threshold.

- Not surprisingly, the interface to the tissue is important. Present electrode arrays for cochlear implants do not support more than four to eight functional channels, even though the number of stimulating electrodes is higher than that. Overlapping excitation fields from different electrodes almost certainly degrade their independence.

- Interlacing of stimulus pulses across electrodessuch that only one electrode is active at any one time- - has proved to be highly effective for cochlear implants in achieving the present levels of electrode and channel independence. Further increases in channel independence (and the number of functional channels) may be achieved through novel electrode designs, placements of electrodes in close proximity to the target neurons, drug treatments to encourage the growth of neural tissue toward electrodes, interlacing of stimuli across bilateral implants, or combinations of these.

- Current processing strategies in widespread useincluding the CIS, HiRes, and ACE strategies for examples-present envelope information but perhaps only a relatively small amount of FS information. Efforts are underway to provide more of the latter information, which, if successful, may be especially helpful for music reception and for speech reception in competition with noise or other talkers.

- Any residual function should be preserved and used to the maximum extent possible in conjunction with the prosthesis, as in combined EAS of the auditory system for persons with some residual (low-frequency) hearing.

- Electrical stimulation of both ears with bilateral cochlear implants also makes better use of what remains in both the peripheral and central auditory systems and can confer large benefits compared with stimulation on one side only.

- High variability in outcomes remains a principal problem to be solved with cochlear implants. Persons using the same speech processor, transcutaneous link, and implanted electronics and electrodes may have 
results ranging from the floor to the ceiling for difficult tests such as the recognition of monosyllabic words. Why this is the case is perhaps the single mostimportant question in current research on implants.

- Good results take time. Asymptotic performance is not achieved with cochlear implants until at least 3 months of daily use and in many cases longer or much longer than that. This and other findings indicate a principal role of the brain in determining outcomes with implants. The time required for asymptomatic performance also indicates that results from acute studies may be misleading in that they may grossly underestimate the potential of an approach.

- A sensory prosthesis and the brain are "partners" in an overall system, and simply focusing on the periphery in the design of a prosthesis may provide good results for persons with fully intact brains and sensory pathways but probably will limit results for persons with impaired pathways or impaired or altered cortical processing.

- The amount of information from the periphery that can be used may be increased through plastic changes in the brain, especially for infants and very young children but also for older patients, albeit at a likely slower pace of adaptation and perhaps to a lesser extent than with young children.

- Desired plastic changes may be facilitated and augmented through directed training; the optimal training procedure is likely to vary according to the age of the patient, the duration of sensory deprivation prior to the restoration of (some) function with a cochlear implant (or bilateral cochlear implants), and whether or not the patient's hearing was first lost prior to the "sensitive period" for the normal development of the auditory pathways and processing in the midbrain and cortex. Training may or may not be effective for patients who lost their hearing prior to or during the sensitive period and had it reinstated (at least to some extent) after the sensitive period had expired. Training may be most effective for persons who lost the sense following the sensitive period and after the sensory pathways and associated cortical processing had been established.

- The highly deleterious effects of cross-modal plasticity or missing the sensitive period for maturation of the central auditory pathways and cortex are "moral imperatives" to screen infants for deafness or hearing impairments and to provide at least some input to the "auditory brain" if feasible and as soon as possible for cases in which severe deficits are found.

- Cochlear implants work as a system, in which all parts are important, including the microphone, the processing strategy, the transcutaneous link, the receiver/stimulator, the implanted electrodes, the functional anatomy of the implanted cochlea, and the user's brain. Among these, the brain has received the least attention in implant designs to date.

- The future of cochlear implants is bright, with multiple outstanding possibilities for even higher levels of performance.

\section{ACKNOWLEDGMENTS}

This article is dedicated to William F. House, MD, whose pioneering work and perseverance provided the foundation and inspiration for subsequent developments. He is the "father" of neuro-otology, and present-day cochlear implants would not have been possible without him.

We thank Prof Richard Tyler and an anonymous reviewer for their thoughtful comments, and we thank Prof Tyler especially for his many suggestions to help us improve an earlier version of this article.

Some of the findings and thoughts in this article were first presented by Prof Wilson in an invited lecture at the 2004 Neural Interfaces Workshop in Bethesda, MD, November 15-17, 2004; in addresses as the Guest of Honor at the Ninth International Conference on Cochlear Implants and Related Sciences in Vienna, Austria, June 14-17, 2006, and at the Sixth Wullstein Symposium 2006: New Developments in Hearing Technology in Würzburg, Germany, December 7-10, 2006; and in a keynote speech at the International Workshop on Advances in Audiology in Salamanca, Spain, May 25-26, 2007. Material was also drawn or adapted from several recent publications by the authors.

Prof Wilson recently became the Chief Strategy Advisor for MED-EL Medical Electronics GmbH, one of the three major cochlear implant companies. None of the statements made in this article favor that or any other company. MED-EL Medical Electronics GmbH did not have any involvement in the study design; data collection, analysis, or interpretation; and writing or submission of this article. (Prof Wilson also serves in multiple other capacities that are not related to his consulting role with MED-EL.) 
This material was based on work supported in part by NIH project N01-DC-2-1002 (to BSW) and its predecessors, all titled "Speech processors for auditory prostheses"; and by NIH project 5R01DC000654 (to MFD) and its predecessors, all titled "Auditory function and speech perception with cochlear implants.”

The authors have declared that no competing interests exist.

\section{REFERENCES}

1. National Institutes of Health Consensus Conference. Cochlear implants in adults and children. JAMA. 1995; 274(24):1955-61. [PMID: 8568992]

2. Eisen MD. History of the cochlear implant. In: Waltzman SB, Roland JT Jr, editors. Cochlear implants. 2nd ed. New York (NY): Thieme; 2006. p. 1-10.

3. Finn R, Hudspeth AJ, Zwislocki J, Young E, Merzenich M. Sound from silence: The development of cochlear implants. In: Beyond discovery: The path from research to human benefit. Washington (DC): National Academy of Sciences; 1999. p. 1-8.

4. Niparko JK, Wilson BS. History of cochlear implants. In: Niparko JK, Kirk KI, Mellon NK, Robbins AM, Tucci DL, Wilson BS, editors. Cochlear implants: Principles \& practices. Philadelphia (PA): Lippincott Williams \& Wilkins; 2000. p. 103-8.

5. Wilson BS, Dorman MF. Interfacing sensors with the nervous system: Lessons from the development and success of the cochlear implant. IEEE Sensors J. 2008;8(1):131-47.

6. Hinojosa R, Marion M. Histopathology of profound sensorineural deafness. Ann N Y Acad Sci. 1983;405:459-84. [PMID: 6575668]

7. Miura M, Sando I, Hirsch BE, Orita Y. Analysis of spiral ganglion cell populations in children with normal and pathological ears. Ann Otol Rhinol Laryngol. 2002; 111(12 Pt 1):1059-65. [PMID: 12498365$]$

8. Leake PA, Rebscher SJ. Anatomical considerations and long-term effects of electrical stimulation. In: Zeng FG, Popper AN, Fay RR, editors. Auditory prostheses: Cochlear implants and beyond. New York (NY): Springer-Verlag; 2004. p. 101-48.

9. Lawson DT, Wilson BS, Zerbi M, Finley CC. Speech processors for auditory prostheses. Third Quarterly Progress Report, NIH project N01-DC-5-2103. Bethesda (MD): Neural Prosthesis Program, National Institutes of Health; 1996.

10. Fishman KE, Shannon RV, Slattery WH. Speech recognition as a function of the number of electrodes used in the
SPEAK cochlear implant speech processor. J Speech Lang Hear Res. 1997;40(5):1201-15. [PMID: 9328890]

11. Wilson BS. The future of cochlear implants. Br J Audiol. 1997;31(4):205-25. [PMID: 9307818]

12. Kiefer J, Von Ilberg C, Rupprecht V, Hubner-Egener J, Knecht R. Optimized speech understanding with the continuous interleaved sampling speech coding strategy in patients with cochlear implants: Effect of variations in stimulation rate and number of channels. Ann Otol Rhinol Laryngol. 2000;109(11):1009-20. [PMID: 11089991]

13. Friesen LM, Shannon RV, Baskent D, Wang X. Speech recognition in noise as a function of the number of spectral channels: Comparison of acoustic hearing and cochlear implants. J Acoust Soc Am. 2001;110(2):1150-63. [PMID: 11519582]

14. Garnham C, O’Driscoll M, Ramsden And R, Saeed S. Speech understanding in noise with a Med-El COMBI 40+ cochlear implant using reduced channel sets. Ear Hear. 2002;23(6):540-52. [PMID: 12476091]

15. Cohen LT, Saunders E, Knight MR, Cowan RS. Psychophysical measures in patients fitted with Contour and straight Nucleus electrode arrays. Hear Res. 2006;212(1-2):160-75. [PMID: 16403611]

16. Frijns JH, Briaire JJ, Grote JJ. The importance of human cochlear anatomy for the results of modiolus-hugging multichannel cochlear implants. Otol Neurotol. 2001;22(3): 340-49. [PMID: 11347637]

17. Gstöettner WK, Adunka O, Franz P, Hamzavi J Jr, Plenk H Jr, Susani M, Baumgartner W, Kiefer J. Perimodiolar electrodes in cochlear implant surgery. Acta Otolaryngol. 2001;121(2):216-19. [PMID: 11349782]

18. Balkany TJ, Eshraghi AA, Yang N. Modiolar proximity of three perimodiolar cochlear implant electrodes. Acta Otolaryngol. 2002;122(4):363-69. [PMID: 12125990]

19. Arts HA, Jones DA, Anderson DJ. Prosthetic stimulation of the auditory system with intraneural electrodes. Ann Otol Rhinol Laryngol Suppl. 2003;191:20-25. [PMID: 14533840]

20. Badi AN, Kertesz TR, Gurgel RK, Shelton C, Normann RA. Development of a novel eighth-nerve intraneural auditory neuroprosthesis. Laryngoscope. 2003;113(5):833-42. [PMID: 12792319]

21. Hillman T, Badi AN, Normann RA, Kertesz T, Shelton C. Cochlear nerve stimulation with a 3-dimensional penetrating electrode array. Otol Neurotol. 2003;24(5):764-68. [PMID: 14501454]

22. Wilson BS. Engineering design of cochlear implants. In: Zeng FG, Popper AN, Fay RR, editors. Auditory prostheses: Cochlear implants and beyond. New York (NY): Springer; 2004. p. 14-52. 
23. Spelman FA. Cochlear electrode arrays: Past, present and future. Audiol Neurootol. 2006;11(2):77-85.

[PMID: 16439830]

24. Badi AN, Owa AO, Shelton C, Normann RA. Electrode independence in intraneural cochlear nerve stimulation. Otol Neurotol. 2007;28(1):16-24. [PMID: 17195741]

25. Middlebrooks JC, Snyder RL. Auditory prosthesis with a penetrating nerve array. J Assoc Res Otolaryngol. 2007; 8(2):258-79. [PMID: 17265124]

26. Wilson BS, Finley CC, Lawson DT, Wolford RD, Eddington DK, Rabinowitz WM. Better speech recognition with cochlear implants. Nature. 1991;352(6332):236-38. [PMID: 1857418$]$

27. Wilson BS, Finley CC, Farmer JC Jr, Lawson DT, Weber BA, Wolford RD, Kenan PD, White MW, Merzenich MM, Schindler RA. Comparative studies of speech processing strategies for cochlear implants. Laryngoscope. 1988;98(10):1069-77. [PMID: 3172953]

28. Skinner MW, Clark GM, Whitford LA, Seligman PM, Staller SJ, Shipp DB, Shallop JK, Everingham C, Menapace CM, Arndt PL, et al. Evaluation of a new spectral peak coding strategy for the Nucleus 22 Channel Cochlear Implant System. Am J Otol. 1994;15 Suppl 2:15-27.

[PMID: 8572106]

29. Kiefer J, Hohl S, Stürzebecher E, Pfennigdorff T, Gstöettner W. Comparison of speech recognition with different speech coding strategies (SPEAK, CIS, and ACE) and their relationship to telemetric measures of compound action potentials in the Nucleus CI 24M cochlear implant system. Audiology. 2001;40(1):32-42. [PMID: 11296939]

30. Koch DB, Osberger MJ, Segal P, Kessler D. HiResolution and conventional sound processing in the HiResolution bionic ear: Using appropriate outcome measures to assess speech recognition ability. Audiol Neurootol. 2004;9(4): 214-23. [PMID: 15205549]

31. Wilson BS. Speech processing strategies. In: Cooper H, Craddock LC, editors. Cochlear implants: A practical guide. 2nd ed. London (England): Whurr; 2006. p. 21-69.

32. Nie K, Stickney G, Zeng FG. Encoding frequency modulation to improve cochlear implant performance in noise. IEEE Trans Biomed Eng. 2005;52(1):64-73. [PMID: 15651565]

33. Wilson BS, Schatzer R, Lopez-Poveda EA, Sun X, Lawson DT, Wolford RD. Two new directions in speech processor design for cochlear implants. Ear Hear. 2005;26 (4 Suppl):73S-81S. [PMID: 16082269]

34. Zeng FG, Nie K, Stickney GS, Kong YY, Vongphoe M, Bhargave A, Wei C, Cao K. Speech recognition with amplitude and frequency modulations. Proc Natl Acad Sci U S A. 2005;102(7):2293-98. [PMID: 15677723]

35. Hochmair I, Nopp P, Jolly C, Schmidt M, Schösser H, Garnham C, Anderson I. MED-EL Cochlear implants:
State of the art and a glimpse into the future. Trends Amplif. 2006;10(4):201-19. [PMID: 17172548]

36. Arnoldner C, Riss D, Brunner M, Durisin M, Baumgartner WD, Hamzavi JS. Speech and music perception with the new fine structure speech coding strategy: Preliminary results. Acta Otolaryngol. 2007;127(12):1298-1303. [PMID: 17851892]

37. Hilbert D. Grundzüge einer allgemeinen Theorie der linearen Integralgleichungen. Leipzig: Teubner; 1912.

38. Smith ZM, Delgutte B, Oxenham AJ. Chimaeric sounds reveal dichotomies in auditory perception. Nature. 2002; 416(6876):87-90. [PMID: 11882898]

39. Zeng FG. Temporal pitch in electric hearing. Hear Res. 2002;174(1-2):101-6. [PMID: 12433401]

40. Baumann U, Nobbe A. Pulse rate discrimination with deeply inserted electrode arrays. Hear Res. 2004;196(1-2):49-57. [PMID: 15464301]

41. Wilson BS, Lawson DT, Finley CC, Zerbi M. Speech processors for auditory prostheses: Randomized update orders; slow rate CIS implementations; channel number manipulations; evaluation of other promising processing strategies; performance of CIS and CA processors in noise; and use and possible development of new test materials. Tenth Quarterly Progress Report, NIH project N01-DC-9-2401. Bethesda (MD): Neural Prosthesis Program, National Institutes of Health; 1991.

42. Green T, Faulkner A, Rosen S. Enhancing temporal cues to voice pitch in continuous interleaved sampling cochlear implants. J Acoust Soc Am. 2004;116(4 Pt 1):2298-2310. [PMID: 15532661]

43. Wilson BS, Lawson DT, Zerbi M, Finley CC. Speech processors for auditory prostheses: Virtual channel interleaved sampling (VCIS) processors-Initial studies with subject SR2. First Quarterly Progress Report, NIH project N01-DC-2-2401. Bethesda (MD): Neural Prosthesis Program, National Institutes of Health; 1992.

44. Wilson BS, Zerbi M, Lawson DT. Speech processors for auditory prostheses: Identification of virtual channels on the basis of pitch. Third Quarterly Progress Report, NIH project N01-DC-2-2401. Bethesda (MD): Neural Prosthesis Program, National Institutes of Health; 1993.

45. Wilson BS, Lawson DT, Zerbi M. Speech processors for auditory prostheses: Evaluation of VCIS processors. Sixth Quarterly Progress Report, NIH project N01-DC-2-2401. Bethesda (MD): Neural Prosthesis Program, National Institutes of Health; 1994.

46. Wilson BS, Lawson DT, Zerbi M, Finley CC. Recent developments with the CIS strategies. In: HochmairDesoyer I, Hochmair ES, editors. Advances in cochlear implants: Proceedings of the 3rd International Cochlear Implant Conference; 1993 Apr; Vienna (Austria). Wien (Austria): Manz; 1994. p. 103-12. 
47. Poroy O, Loizou PC. Pitch perception using virtual channels. In: Proceedings of the 2001 Conference on Implantable Auditory Prostheses; 2001 Aug 19-24; Pacific Grove (CA).

48. Litvak LM, Overstreet E, Mishra L. Steering current through simultaneous activation of intracochlear electrodes in the Clarion CII cochlear implant: Frequency resolution. In: Proceedings of the 2003 Conference on Implantable Auditory Prostheses; 2003 Aug 17-22; Pacific Grove (CA).

49. Donaldson GS, Kreft HA, Litvak L. Place-pitch discrimination of single- versus dual-electrode stimuli by cochlear implant users (L). J Acoust Soc Am. 2005;118(2):623-26. [PMID: 16158620]

50. Firszt JB, Koch DB, Downing M, Litvak L. Current steering creates additional pitch percepts in adult cochlear implant recipients. Otol Neurotol. 2007;28(5):629-36. [PMID: 17667771]

51. Koch DB, Downing M, Osberger MJ, Litvak L. Using current steering to increase spectral resolution in CII and HiRes 90K users. Ear Hear. 2007;28(2 Suppl):39S-41S. [PMID: 17496643]

52. Wilson BS, Schatzer R, Lopez-Poveda EA. Possibilities for a closer mimicking of normal auditory functions with cochlear implants. In: Waltzman SB, Roland JT Jr, editors. Cochlear implants. 2nd ed. New York (NY): Thieme; 2006. p. 48-56.

53. McDermott HJ, McKay CM. Pitch ranking with nonsimultaneous dual electrode electrical stimulation of the cochlea. J Acoust Soc Am. 1994;96(1):155-62.

[PMID: 8064018$]$

54. Kwon BJ, Van den Honert C. Dual-electrode pitch discrimination with sequential interleaved stimulation by cochlear implant users. J Acoust Soc Am. 2006;120(1): EL1-6. [PMID: 16875252]

55. Nobbe A, Schleich P, Zierhofer C, Nopp P. Frequency discrimination with sequential or simultaneous stimulation in MED-EL cochlear implants. Acta Otolaryngol. 2007; 127(12):1266-72. [PMID: 17851932]

56. Spahr AJ, Dorman MF, Loiselle LH. Performance of patients using different cochlear implant systems: Effects of input dynamic range. Ear Hear. 2007;28(2):260-75. [PMID: 17496675]

57. Helms J, Müller J, Schön F, Moser L, Arnold W, Janssen T, Ramsden R, Von Ilberg C, Kiefer J, Pfennigdorf T, Gstöttner W, Baumgartner W, Ehrenberger K, Skarzynski H, Ribari O, Thumfart W, Stephan K, Mann W, Heinemann M, Zorowka P, Lippert KL, Zenner HP, Bohndord M, Hüttenbrink K, Hochmair-Desoyer I, et al. Evaluation of performance with the COMBI40 cochlear implant in adults: A multicentric clinical study. ORL J Otorhinolaryngol Relat Spec. 1997;59(1):23-35. [PMID: 9104746]
58. Wilson BS, Dorman MF. The surprising performance of present-day cochlear implants. IEEE Trans Biomed Eng. 2007;54(6 Pt 1):969-72. [PMID: 17554816]

59. Frijns JH, Briaire JJ, De Laat JA, Grote JJ. Initial evaluation of the Clarion CII cochlear implant: Speech perception and neural response imaging. Ear Hear. 2002; 23(3):184-97. [PMID: 12072611]

60. Dorman MF, Loizou PC, Fitzke J, Tu Z. Recognition of monosyllabic words by cochlear implant patients and by normal-hearing subjects listening to words processed through cochlear implant signal processing strategies. Ann Otol Rhinol Laryngol Suppl. 2000;185:64-66. [PMID: 11141009]

61. Van den Honert C, Stypulkowski PH. Single fiber mapping of spatial excitation patterns in the electrically stimulated auditory nerve. Hear Res. 1987;29(2-3):195-206. [PMID: 3624083$]$

62. Fu QJ. Temporal processing and speech recognition in cochlear implant users. Neuroreport. 2002;13(13):1635-39. [PMID: 12352617]

63. Wilson BS, Lawson DT, Müller JM, Tyler RS, Kiefer J. Cochlear implants: Some likely next steps. Annu Rev Biomed Eng. 2003;5:207-49. [PMID: 12704085]

64. Robles L, Ruggero MA. Mechanics of the mammalian cochlea. Physiol Rev. 2001;81(3):1305-52. [PMID: 11427697]

65. Dallos P. The active cochlea. J Neurosci. 1992;12(12): 4575-85. [PMID: 1464757]

66. Smith RL. Cochlear processes reflected in responses of the cochlear nerve. Acta Otolaryngol. 1985;100(1-2):1-12. [PMID: 2992224]

67. Guinan JJ Jr. Physiology of olivocochlear efferents. In: Dallos P, Popper AN, Fay RR, editors. The cochlea. New York (NY): Springer; 1996. p. 435-502.

68. Kiang NY, Guinan JJ Jr, Liberman MC, Brown MC, Eddington DK. Feedback control mechanisms of the auditory periphery: Implications for cochlear implants. In: Banfai P, editor. Cochlear implant: Current situation. Erkelenz (Germany): Bermann; 1988. p. 131-51.

69. Liberman MC. Auditory-nerve response from cats raised in a low-noise chamber. J Acoust Soc Am. 1978;63(2): 442-55. [PMID: 670542]

70. Kiang NY, Watanabe T, Thomas EC, Clark LF. Discharge patterns of single fibers in the cat's auditory nerve. Cambridge (MA): MIT Press; 1965.

71. Hartmann R, Topp G, Klinke R. Discharge patterns of cat primary auditory nerve fibers with electrical stimulation of the cochlea. Hear Res. 1984;13(1):47-62. [PMID: 6546751]

72. Kiang NYS, Moxon EC, Levine RA. Auditory-nerve activity in cats with normal and abnormal cochleas. In: Wolstenholme GE, Knight J, editors. Sensorineural hearing loss. London (England): Churchill; 1970. p. 241-73. 
73. Shepherd RK, Javel E. Electrical stimulation of the auditory nerve. I. Correlation of physiological responses with cochlear status. Hear Res. 1997;108(1-2):112-44. [PMID: 9213127]

74. Blamey P, Arndt P, Bergeron F, Bredberg G, Brimacombe J, Facer G, Larky J, Lindström B, Nedzelski J, Peterson A, Shipp D, Staller S, Whitford L. Factors affecting auditory performance of postlinguistically deaf adults using cochlear implants. Audiol Neurootol. 1996;1(5):293-306. [PMID: 9390810]

75. Shepherd RK, Hardie NA. Deafness-induced changes in the auditory pathway: Implications for cochlear implants. Audiol Neurootol. 2001;6(6):305-18. [PMID: 11847461]

76. Lee DS, Lee JS, Oh SH, Kim SK, Kim JW, Chung JK, Lee MC, Kim CS. Cross-modal plasticity and cochlear implants. Nature. 2001;409(6817):149-50. [PMID: 11196628]

77. Bavelier D, Neville HJ. Cross-modal plasticity: Where and how? Nat Rev Neurosci. 2002;3(6):443-52. [PMID: 12042879]

78. Nadol JB Jr, Shiao JY, Burgess BJ, Ketten DR, Eddington DK, Gantz BJ, Kos I, Montandon P, Coker NJ, Roland JT Jr, Shallop JK. Histopathology of cochlear implants in humans. Ann Otol Rhinol Laryngol. 2001;110(9):883-91. [PMID: 11558767]

79. Khan AM, Handzel O, Burgess BJ, Damian D, Eddington DK, Nadol JB Jr. Is word recognition correlated with the number of surviving spiral ganglion cells and electrode insertion depth in human subjects with cochlear implants? Laryngoscope. 2005;115(4):672-77. [PMID: 15805879]

80. Fayad JN, Linthicum FH Jr. Multichannel cochlear implants: Relation of histopathology to performance. Laryngoscope. 2006;116(8):1310-20. [PMID: 16885730]

81. Blamey P. Are spiral ganglion cell numbers important for speech perception with a cochlear implant? Am J Otol. 1997; 18(6 Suppl):S11-12. [PMID: 9391577]

82. Sharma A, Dorman MF, Spahr AJ. Rapid development of cortical auditory evoked potentials after early cochlear implantation. Neuroreport. 2002;13(10):1365-68. [PMID: 12151804]

83. Dorman MF, Wilson BS. The design and function of cochlear implants: Human speech can be transformed into an electrical code that deafened ears can "hear." Am Scientist. 2004;92(5):436-45.

84. Dorman MF, Spahr AJ. Speech perception by adults with multichannel cochlear implants. In: Waltzman SB, Roland JT Jr, editors. Cochlear implants. 2nd ed. New York (NY): Thieme; 2006. p. 193-204.

85. Dorman MF, Loizou PC, Spahr AJ, Maloff E. A comparison of the speech understanding provided by acoustic models of fixed-channel and channel-picking signal processors for cochlear implants. J Speech Lang Hear Res. 2002;45(4):783-88. [PMID: 12199407]
86. Shannon RV, Fu QJ, Galvin J 3rd. The number of spectral channels required for speech recognition depends on the difficulty of the listening situation. Acta Otolaryngol Suppl. 2004;(552):50-54. [PMID: 15219048]

87. Fu QJ, Nogaki G. Noise susceptibility of cochlear implant users: The role of spectral resolution and smearing. J Assoc Res Otolaryngol. 2005;6(1):19-27. [PMID: 15735937]

88. Ranck JB Jr. Which elements are excited in electrical stimulation of mammalian central nervous system: A review. Brain Res. 1975;98(3):417-40. [PMID: 1102064$]$

89. Roehm PC, Hansen MR. Strategies to preserve or regenerate spiral ganglion neurons. Curr Opin Otolaryngol Head Neck Surg. 2005;13(5):294-300. [PMID: 16160524

90. Pettingill LN, Richardson RT, Wise AK, O’Leary SJ, Shepherd RK. Neurotrophic factors and neural prostheses: Potential clinical applications based upon findings in the auditory system. IEEE Trans Biomed Eng. 2007;54(6 Pt 1):1138-48. [PMID: 17551571]

91. Rejali D, Lee VA, Abrashkin KA, Humayun N, Swiderski DL, Rapheal Y. Cochlear implants and ex vivo BDNF gene therapy protect spiral ganglion neurons. Hear Res. 2007;228(1-2):180-87. [PMID: 17416474]

92. Vieira M, Christensen BL, Wheeler BC, Feng AS, Kollmar R. Survival and stimulation of neurite outgrowth in a serum-free culture of spiral ganglion neurons from adult mice. Hear Res. 2007;230(1-2):17-23. [PMID: 17521837]

93. Von Ilberg C, Kiefer J, Tillein J, Pfenningdorff T, Hartmann R, Stürzebecher E, Klinke R. Electric-acoustic stimulation of the auditory system. New technology for severe hearing loss. ORL J Otorhinolaryngol Relat Spec. 1999; 61(6):334-40. [PMID: 10545807]

94. Kiefer J, Tillein J, Von Ilberg C, Pfennigdorff T, Stürzebecher E, Klinke R, Gstöettner W. Fundamental aspects and first results of the clinical application of combined electric and acoustic stimulation of the auditory system. In: Kubo T, Takahashi Y, Iwaki T, editors. Cochlear implants: An update. The Hague (the Netherlands): Kugler Publications; 2002. p. 569-76.

95. Skarzyski H, Lorens A, D’Haese P, Walkowiak A, Piotrowska A, Sliwa L, Anderson I. Preservation of residual hearing in children and post-lingually deafened adults after cochlear implantation: An initial study. ORL J Otorhinolaryngol Relat Spec. 2002;64(4):247-53.

[PMID: 12232469]

96. Gantz BJ, Turner CW. Combining acoustic and electrical hearing. Laryngoscope. 2003;113(10):1726-30.

[PMID: 14520097]

97. Gstöettner W, Kiefer J, Baumgartner WD, Pok S, Peters S, Adunka O. Hearing preservation in cochlear implantation for electric acoustic stimulation. Acta Otolaryngol. 2004;124(4):348-52. [PMID: 15224851] 
98. Kiefer J, Gstöettner W, Baumgartner W, Pok SM, Tillein J, Ye Q, Von Ilberg C. Conservation of low-frequency hearing in cochlear implantation. Acta Otolaryngol. 2004; 124(3):272-80. [PMID: 15141755]

99. Gantz BJ, Turner C, Gfeller KE, Lowder MW. Preservation of hearing in cochlear implant surgery: Advantages of combined electrical and acoustical speech processing. Laryngoscope. 2005;115(5):796-802. [PMID: 15867642]

100. James C, Albegger K, Battmer R, Burdo S, Deggouj N, Dequine O, Dillier N, Gersdorff M, Laszig R, Lenarz T, Rodriguez MM, Mondain M, Offeciers E, Macías AR, Ramsden R, Sterkers O, Von Wallenberg E, Weber B, Fraysse B. Preservation of residual hearing with cochlear implantation: How and why. Acta Otolaryngol. 2005; 125(5):481-91. [PMID: 16092537]

101. Kiefer J, Pok M, Adunka O, Stürzebecher E, Baumgartner W, Schmidt M, Tillein J, Ye Q, Gstöettner W. Combined electric and acoustic stimulation of the auditory system: Results of a clinical study. Audiol Neurootol. 2005;10(3): 134-44. [PMID: 15724084]

102. Fraysse B, Macías AR, Sterkers O, Burdo S, Ramsden R, Deguine O, Klenzner T, Lenarz T, Rodriguez MM, Von Wallenberg E, James C. Residual hearing conservation and electroacoustic stimulation with the nucleus 24 contour advance cochlear implant. Otol Neurotol. 2006;27(5): 624-33. [PMID: 16868510]

103. Gantz BJ, Turner C, Gfeller KE. Acoustic plus electric speech processing: Preliminary results of a multicenter clinical trial of the Iowa/Nucleus Hybrid implant. Audiol Neurootol. 2006;11Suppl 1:63-68. [PMID: 17063013]

104. Gstöettner WK, Helbig S, Maier N, Kiefer J, Radeloff A, Adunka OF. Ipsilateral electric acoustic stimulation of the auditory system: Results of long-term hearing preservation. Audiol Neurootol. 2006;11 Suppl 1:49-56. [PMID: 17063011]

105. Skarzynski H, Lorens A, Piotrowska A, Anderson I. Partial deafness cochlear implantation provides benefit to a new population of individuals with hearing loss. Acta Otolaryngol. 2006;126(9):934-40. [PMID: 16864490]

106. Skarzynski H, Lorens A, Piotrowska A, Anderson I. Preservation of low frequency hearing in partial deafness cochlear implantation (PDCI) using the round window surgical approach. Acta Otolaryngol. 2007;127(1):41-48. [PMID: 17364328]

107. Ye Q, Tillein J, Hartmann R, Gstöettner W, Kiefer J. Application of a corticosteroid (Triamcinolon) protects inner ear function after surgical intervention. Ear Hear. 2007;28(3):361-69. [PMID: 17485985]

108. Adunka O, Unkelbach MH, Mack M, Hambek M, Gstöettner W, Kiefer J. Cochlear implantation via the round window membrane minimizes trauma to cochlear structures:
A histologically controlled insertion study. Acta Otolaryngol. 2004;124(7):807-12. [PMID: 15370564]

109. Adunka O, Gstöettner W, Hambek M, Unkelbach $\mathrm{MH}$, Radeloff A, Kiefer J. Preservation of basal inner ear structures in cochlear implantation. ORL J Otorhinolaryngol Relat Spec. 2004;66(6):306-12. [PMID: 15668529]

110. Lehnhardt E. Intracochlear placement of cochlear implant electrodes in soft surgery technique [German]. HNO. 1993;41(7):356-59. [PMID: 8376183]

111. Lawson DT, Brill S, Wolford RD, Wilson BS, Schatzer R. Speech processors for auditory prostheses: Binaural cochlear implant findings-Summary of initial studies with eleven subjects. Ninth Quarterly Progress Report, NIH project N01-DC-8-2105. Bethesda (MD): Neural Prosthesis Program, National Institutes of Health; 2000.

112. Lawson DT, Wolford RD, Brill SM, Schatzer R, Wilson BS. Speech processors for auditory prostheses: Further studies regarding benefits of bilateral cochlear implants. Twelfth Quarterly Progress Report, NIH project N01-DC8-2105. Bethesda (MD): Neural Prosthesis Program, National Institutes of Health; 2001.

113. Gantz BJ, Tyler RS, Rubinstein JT, Wolaver A, Lowder M, Abbas P, Brown C, Hughes M, Preece JP. Binaural cochlear implants placed during the same operation. Otol Neurotol. 2002;23(2):169-80. [PMID: 11875346]

114. Müller J, Schön F, Helms J. Speech understanding in quiet and noise in bilateral users of the MED-EL COMBI 40/40+ cochlear implant system. Ear Hear. 2002;23(3):198-206. [PMID: 12072612]

115. Schön F, Müller J, Helms J. Speech reception thresholds obtained in a symmetrical four-loudspeaker arrangement from bilateral users of MED-EL cochlear implants. Otol Neurotol. 2002;23(5):710-14. [PMID: 12218624]

116. Tyler RS, Gantz BJ, Rubinstein JT, Wilson BS, Parkinson AJ, Wolaver A, Preece JP, Witt S, Lowder MW. Threemonth results with bilateral cochlear implants. Ear Hear. 2002;23(1 Suppl):80S-89S. [PMID: 11883771]

117. Van Hoesel R, Ramsden R, O’Driscoll M. Sound-direction identification, interaural time delay discrimination, and speech intelligibility advantages in noise for a bilateral cochlear implant user. Ear Hear. 2002;23(2):137-49. [PMID: 11951849]

118. Tyler RS, Dunn CC, Witt SA, Preece JP. Update on bilateral cochlear implantation. Curr Opin Otolaryngol Head Neck Surg. 2003;11(5):388-93. [PMID: 14502072]

119. Van Hoesel RJ, Tyler RS. Speech perception, localization, and lateralization with bilateral cochlear implants. J Acoust Soc Am. 2003;113(3):1617-30. [PMID: 12656396]

120. Laszig R, Aschendorff A, Stecker M, Müller-Deile J, Maune S, Dillier N, Weber B, Hey M, Begall K, Lenarz T, Battmer RD, Böhm M, Steffens T, Strutz J, Linder T, Probst R, Allum J, Westhofen M, Doering W. Benefits of 
WILSON and DORMAN. Cochlear implants: Current designs and future possibilities

bilateral electrical stimulation with the nucleus cochlear implant in adults: 6-month postoperative results. Otol Neurotol. 2004;25(6):958-68. [PMID: 15547426]

121. Schleich P, Nopp P, D’Haese P. Head shadow, squelch, and summation effects in bilateral users of the MED-EL COMBI 40/40+ cochlear implant. Ear Hear. 2004;25(3): 197-204. [PMID: 15179111]

122. Das S, Buchman CA. Bilateral cochlear implantation: Current concepts. Curr Opin Otolaryngol Head Neck Surg. 2005;13(5):290-93. [PMID: 16160523]

123. Ramsden R, Greenham P, O’Driscoll M, Mawman D, Proops D, Craddock L, Fielden C, Graham J, Meerton L, Verschuur C, Toner J, McAnallen C, Osborne J, Doran M, Gray R, Pickerill M. Evaluation of bilaterally implanted adult subjects with the Nucleus 24 cochlear implant system. Otol Neurotol. 2005;26(5):988-98. [PMID: 16151348]

124. Senn P, Kompis M, Vischer M, Haeusler R. Minimum audible angle, just noticeable interaural differences and speech intelligibility with bilateral cochlear implants using clinical speech processors. Audiol Neurootol. 2005; 10(6):342-52. [PMID: 16103645]

125. Litovsky RY, Johnstone PM, Godar SP. Benefits of bilateral cochlear implants and/or hearing aids in children. Int J Audiol. 2006;45 Suppl 1:S78-91. [PMID: 16938779]

126. Litovsky R, Parkinson A, Arcaroli J, Sammeth C. Simultaneous bilateral cochlear implantation in adults: A multicenter clinical study. Ear Hear. 2006;27(6):714-31. [PMID: 17086081]

127. Tyler RS, Dunn CC, Witt SA, Noble WG. Speech perception and localization with adults with bilateral sequential cochlear implants. Ear Hear. 2007;28(2 Suppl):86S-90S. [PMID: 17496655]

128. Nopp P, Schleich P, D’Haese P. Sound localization in bilateral users of MED-EL COMBI 40/40+ cochlear implants. Ear Hear. 2004;25(3):205-14. [PMID: 15179112]

129. Seeber BU, Baumann U, Fastl H. Localization ability with bimodal hearing aids and bilateral cochlear implants. J Acoust Soc Am. 2004;116(3):1698-1709. [PMID: 15478437]

130. Schoen F, Mueller J, Helms J, Nopp P. Sound localization and sensitivity to interaural cues in bilateral users of the Med-El Combi 40/40+ cochlear implant system. Otol Neurotol. 2005;26(3):429-37. [PMID: 15891645]

131. Verschuur CA, Lutman ME, Ramsden R, Greenham P, O’Driscoll M. Auditory localization abilities in bilateral cochlear implant recipients. Otol Neurotol. 2005;26(5): 965-71. [PMID: 16151344]

132. Neuman AC, Haravon A, Sislian N, Waltzman SB. Sounddirection identification with bilateral cochlear implants. Ear Hear. 2007;28(1):73-82. [PMID: 17204900]

133. Grantham DW, Ashmead DH, Ricketts TA, Labadie RF, Haynes DS. Horizontal-plane localization of noise and speech signals by postlingually deafened adults fitted with bilateral cochlear implants. Ear Hear. 2007;28(4):524-41. [PMID: 17609614]

134. Wilson BS, Wolford RD, Lawson DT, Schatzer R. Speech processors for auditory prostheses: Additional perspectives on speech reception with combined electric and acoustic stimulation. Third Quarterly Progress Report, NIH project N01-DC-2-1002. Bethesda (MD): Neural Prosthesis Program, National Institutes of Health; 2002.

135. Skarzyski H, Lorens A, Piotrowska A. A new method of partial deafness treatment. Med Sci Monit. 2003;9(4): CS20-24. [PMID: 12709676]

136. Turner CW, Gantz BJ, Vidal C, Behrens A, Henry BA. Speech recognition in noise for cochlear implant listeners: Benefits of residual acoustic hearing. J Acoust Soc Am. 2004;115(4):1729-35. [PMID: 15101651]

137. Kong YY, Stickney GS, Zeng FG. Speech and melody recognition in binaurally combined acoustic and electric hearing. J Acoust Soc Am. 2005;117(3 Pt 1):1351-61. [PMID: 15807023]

138. James CJ, Fraysse B, Deguine O, Lenarz T, Mawman D, Ramos A, Ramsden R, Sterkers O. Combined electroacoustic stimulation in conventional candidates for cochlear implantation. Audiol Neurootol. 2006;11 Suppl 1:57-62. [PMID: 17063012]

139. Gifford RH, Dorman MF, McKarns SA, Spahr AJ. Combined electric and contralateral acoustic hearing: Word and sentence recognition with bimodal hearing. J Speech Lang Hear Res. 2007;50(4):835-43. [PMID: 17675589]

140. Dorman MF, Gifford RH, Spahr AJ, McKarns SA. The benefits of combining acoustic and electric stimulation for the recognition of speech, voice and melodies. Audiol Neurootol. 2008;13(2):105-12. [PMID: 18057874]

141. Dorman MF, Spahr AJ, Loizou PC, Dana CJ, Schmidt JS. Acoustic simulations of combined electric and acoustic hearing (EAS). Ear Hear. 2005;26(4):371-80. [PMID: 16079632]

142. Gfeller KE, Olszewski C, Turner C, Gantz B, Oleson J. Music perception with cochlear implants and residual hearing. Audiol Neurootol. 2006;11 Suppl 1:12-15. [PMID: 17063005]

143. Gfeller K, Turner C, Oleson J, Zhang X, Gantz B, Froman R, Olszewski C. Accuracy of cochlear implant recipients on pitch perception, melody recognition, and speech reception in noise. Ear Hear. 2007;28(3):412-23. [PMID: 17485990]

144. Morera C, Manrique M, Ramos A, Garcia-Ibanez L, Cavalle L, Huarte A, Castillo C, Estrada E. Advantages of binaural hearing provided through bimodal stimulation via a cochlear implant and a conventional hearing aid: A 6-month comparative study. Acta Otolaryngol. 2005; 125(6):596-606. [PMID: 16076708$]$ 
145. Ching TY, Incerti P, Hill M. Binaural benefits for adults who use hearing aids and cochlear implants in opposite ears. Ear Hear. 2004;25(1):9-21. [PMID: 14770014]

146. Tyler RS, Dunn CC, Witt SA, Noble W, Gantz BJ, Rubinstein JT, Parkinson AJ, Branin SC. Soundfield hearing for patients with cochlear implants and hearing aids. In: Cooper H, Craddock LC, editors. Cochlear implants: A practical guide. 2nd ed. London (England): Whurr; 2006. p. 338-66.

147. Qin MK, Oxenham AJ. Effects of simulated cochlearimplant processing on speech reception in fluctuating maskers. J Acoust Soc Am. 2003;114(1):446-54.

[PMID: 12880055]

148. Qin MK, Oxenham AJ. Effects of introducing unprocessed low-frequency information on the reception of envelope-vocoder processed speech. J Acoust Soc Am. 2006;119(4):2417-26. [PMID: 16642854]

149. Spahr AJ, Dorman MF. Performance of subjects fit with the Advanced Bionics CII and Nucleus 3G cochlear implant devices. Arch Otolaryngol Head Neck Surg. 2004;130(5):624-28. [PMID: 15148187]

150. Rubinstein JT, Wilson BS, Finley CC, Abbas PJ. Pseudospontaneous activity: Stochastic independence of auditory nerve fibers with electrical stimulation. Hear Res. 1999; 127(1-2):108-18. [PMID: 9925022]

151. Lawson DT, Wilson BS, Zerbi M, Van den Honert C, Finley CC, Farmer JC Jr, McElveen JT Jr, Roush PA. Bilateral cochlear implants controlled by a single speech processor. Am J Otol. 1998;19(6):758-61. [PMID: 9831150]

152. Linkenhoker BA, Knudsen EI. Incremental training increases the plasticity of the auditory space map in adult barn owls. Nature. 2002;419(6904):293-96.

[PMID: 12239566]

Submitted for publication October 29, 2007. Accepted in revised form March 19, 2008. 